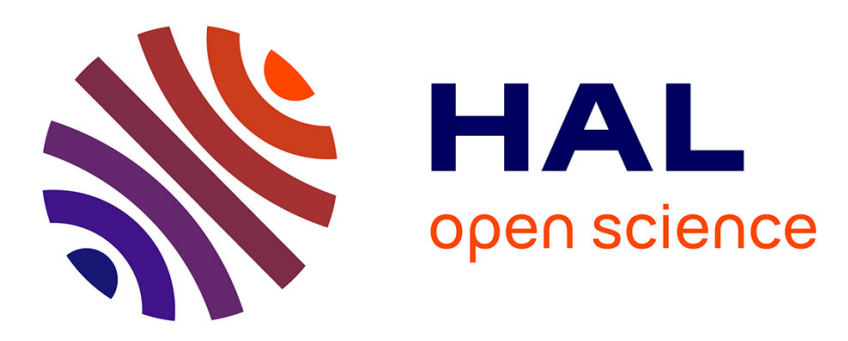

\title{
Wave transmission and reflection analysis through complex media based on the second strain gradient theory
}

Guang Zhu, Abdelmalek Zine, Christophe Droz, Mohamed Ichchou

\section{- To cite this version:}

Guang Zhu, Abdelmalek Zine, Christophe Droz, Mohamed Ichchou. Wave transmission and reflection analysis through complex media based on the second strain gradient theory. European Journal of Mechanics - A/Solids, 2021, 90, pp.104326. 10.1016/j.euromechsol.2021.104326 . hal-03242745

\section{HAL Id: hal-03242745 \\ https://hal.science/hal-03242745}

Submitted on 31 May 2021

HAL is a multi-disciplinary open access archive for the deposit and dissemination of scientific research documents, whether they are published or not. The documents may come from teaching and research institutions in France or abroad, or from public or private research centers.
L'archive ouverte pluridisciplinaire HAL, est destinée au dépôt et à la diffusion de documents scientifiques de niveau recherche, publiés ou non, émanant des établissements d'enseignement et de recherche français ou étrangers, des laboratoires publics ou privés. 


\title{
Wave transmission and reflection analysis through complex media based on the second strain gradient theory
}

\author{
Guang Zhu ${ }^{\mathrm{a}, \mathrm{d}}$, Abdelmalek Zine ${ }^{\mathrm{b}}$, Christophe Droz ${ }^{\mathrm{a}, \mathrm{c}}$, Mohamed Ichchou $^{\mathrm{a}}$ \\ ${ }^{a}$ Vibroacoustics $\mathcal{E}$ Complex Media Research Group, LTDS - CNRS UMR 5513, Centre Lyonnais d'Acoustique CeLyA, École Centrale de Lyon, \\ Université de Lyon, France \\ ${ }^{b}$ Institute Camille Jordan - CNRS UMR 5208, École Centrale de Lyon, France \\ ${ }^{c} K U$ Leuven, Department of Mechanical Engineering, Celestijnenlaan 300, Heverlee, Belgium \\ DMMS-M Lab, Flanders Make, Belgium \\ ${ }^{d}$ Laboratoire Vibrations Acoustique, Centre Lyonnais d'Acoustique CeLyA, INSA Lyon, Université de Lyon, France
}

\begin{abstract}
The scattering of guided waves through a complex structure is of great importance in predicting vibration energy transmission and reflection across structures. Multi-scale models based on generalized continuum theory have been developed recently to investigate the vibration behavior of complex media in the frame of continuum mechanics. In this study, models based on the second strain gradient theory are employed together with a scattering matrix method to study the wave transmission and reflection through non-classical coupling regions between two waveguides. Numerical results are presented for longitudinal and bending waves through 1-D coupling interfaces. Upon which we discuss the local behavior of the internal heterogeneity in the complex coupling region and its impact on wave scattering properties at coupling interfaces. The results obtained also show that the proposed approach is of great potential in the investigation of vibration transmission control by enriching a waveguide's internal structure.
\end{abstract}

Keywords: Second strain gradient theory; complex media; wave scattering; elastic waveguide; transmission coefficient; transmitted energy.

\section{Introduction}

Wave traveling analysis through engineering waveguide structures has raised significant interest over the past decade as it provides an efficient way to describe their vibration behavior, especially in the high-frequency domain. Waveguide structures can be regarded as a combination of individual waveguides connected by different types of joints, thus the analysis of wave propagation in each waveguide and interaction at each coupling joint plays an important role in evaluating and optimizing the structural vibration properties. Many approaches have been developed analytically and numerically for the study of the wave traveling in waveguide structures. Examples of analytical methods include the dynamic stiffness method [1], wave scattering method [2], spectral element method [3, 4], energy flow approach [5, 6], among others. With analytical approaches, the studied structure is often assumed to be isotropic and homogeneous. These assumptions are not true for structures involving complex internal structures such as composites, architectured media and metamaterials. In these complex media, local behavior of the internal heterogeneity has a significant influence on their vibration and wave traveling characteristics. For predicting their vibrational behavior, numerical approaches such as wave finite element methods (WFEM) [7, 8], Spectral Finite Element (SFEM)[9] are developed to calculate the wave propagation characteristics and wave interaction coefficients at complex interfaces.

Considering that numerical simulation often involves large computational resources, especially when the wavelength is approaching the length of spatial period of the system, an alternative solution is developed by enriching the classical continuum theory with the micro-scale behavior explicitly included in the constitutive equation. Accordingly,

\footnotetext{
*mohamed.ichchou@ec-lyon.fr

Email address: mohamed.ichchou@ec-lyon.fr (Mohamed Ichchou)
} 
macroscopic constitutive models that capture the local micro-scale properties can be developed by an appropriate definition of non-local parameters. This theory is known as the generalized continuum theory. Generalized continuum theories were firstly proposed in the 1960s and developed into three different branches in the last few decades: the higher gradient theory, the higher-order theory, and non-local elasticity theory. The basic idea of the generalized continuum theory is to establish a relationship between macroscopic mechanical quantities and microscopic physical quantities within the framework of continuum mechanics.

Generalized continuum theories have been widely utilized in complex media modeling especially in short-wavelength deformation. Based on generalized continuum theories, multi-scale models are developed in recent studies to investigate vibration features of complex media while considering the local behavior of its internal structures, for example, the reformulated beam theories using Eringen's nonlocal differential constitutive relations [10, 11], non-classical Kirchhoff plate model and Mindlin plate model based on Modified Couple Stress (MCS) [12, 13]. Literature shows that the generalized theory-based models are capable of capturing the scale effect of the mechanical behavior observed in the experiments where the specimen characteristic length or the wavelength of the disturbance are comparable to the lengths of the major heterogeneity in the media. The long-range interaction described in generalized theory has significant influences on predicting the non-classical structural characteristics such as deflections, buckling loads, natural frequencies, vibration responses, etc. Recently, the propagation of waves in complex media or nano-scaled structures have been investigated based on generalized continuum theories [14, 15]. Specifically, the analysis often relies on the introduction of a set of characteristic lengths, and it can be observed that the dispersion relation changes substantially in the studied problems from the given type of geometry and loading. The introduced characteristic length parameters by the generalized theory are also crucial to predict non-local wave scattering phenomena [16]. The research of Li et Wei [17, 18] shows the reflection and transmission coefficients at the interface of two dipolar gradient media are dependent on the microstructure parameters along with the incident angular frequency. Mention can also be made of the study proposed by Zhu et al. in 2019 [19], the proposed generalized theory-based rod model predicts that extra evanescent waves are generated along with the dispersive longitudinal wave, and these evanescent waves have a great impact on wave diffusion at a discontinuity between non-local media.

Very seldom research was conducted on the wave conversion and scattering at coupling regions involving nonclassical waveguides. Wang et al did some research on this issue [20], wave scattering through a couple-stress slab sandwiched between two elastic half-spaces was investigated based on the couple stress (CS) theory, the reflection and transmission behaviors are proven to be affected by the couple-stress. Upon this, Liu et al investigated the reflection of elastic waves through the functionally graded piezoelectric microstructures [21]. The employed generalized theories are both CS theory because CS theory is more convenient to use as its constitutive relations are clear with only one additional couple-stress governing the conservation of moment of momentum. However, CS theory is not capable of reflecting all the non-local interaction acting on the micro-body, which leads to that the scattering of transverse waves is sensitive through the CS theory media than the longitudinal wave. Therefore, generalized theories that can capture more complex long-range interaction are more favorable in the wave scattering problem. Furthermore, the wave conversion situation through the non-local media coupled with the classical ones is still not clear, and how the non-local media affects the wave conversion and transmission has not been well investigated either.

To fill this gap, in the present paper Mindlin's Second Strain Gradient (SSG) theory is employed to characterize the non-local inserts between the classical theory (CT) media. SSG theory is developed by introducing the classical strain tensor and the high-order displacement derivatives into the strain energy [22], thus it is capable of capturing more long-distance interactions compared with CS theory and the first strain gradient theory. Despite the complexity of Mindlin's SSG theory (i.e. the introduction of 16 new coefficients) and the difficulty in developing a physical interpretation of these high-order material constants, SSG theory is regarded as the most effective one among the higher gradient theories. How the introduction of the SSG theory-based inserts in a classical theory (CT) based elastic waveguide affects wave conversion and for which benefits are the main focus of this paper. SSG theory-based Timoshenko beam model has been established by Asghari et al.[23], and SSG theory-based rod model by Zhu et al. [19] with Hamilton principle to exploit the structural free-vibration behaviors and wave propagation features. The resulting non-classical wave dispersion relations are employed in the present paper to investigate the wave reflection and transmission properties through a non-classical coupling region with the transfer matrix method (TMM). Predicting the diffusion characteristics of CT - SSG interfaces however requires addressing the coupling between CT and SSG media, each exhibiting different wave types. There is no existing work relating these high-order material properties to wave scattering features. Especially, no investigation was done on the scattering effects produced by a coupling 
region with non-local elasticity within a classical elastic structure. In the present paper, an original relationship is introduced to link the higher-order material properties and non-classical wave scattering characteristics by developing an equivalence concerning all the generalized displacements and generalized forces based on TMM.

The paper is organized as follows: the SSG theory is reviewed in Section 2. The SSG theory-based wave-scattering matrix approach for predicting the diffusion properties of the longitudinal wave through a 1-D complex coupling region between two classical waveguides is presented in Section 3 . Section 4 treats the case of bending wave scattering problem through the complex coupling region. Numerical examples and result discussion are displayed in Section 5 , and conclusions for this work are drawn in Section 6

\section{Review of the SSG theory}

'Second Strain Gradient theory' (SSG theory) was developed by Mindlin by introducing the second and third derivatives of displacement into the strain energy density along with the classical strain tensor. For second strain gradient continua, the strain energy density $\bar{u}$ is written in a quadratic form as [22],

$$
\begin{aligned}
\bar{u}= & \frac{1}{2} \lambda \varepsilon_{i i} \varepsilon_{j j}+\mu \varepsilon_{i j} \varepsilon_{i j}+a_{1} \eta_{i j j} \eta_{i k k}+a_{2} \eta_{i i k} \eta_{k j j}+a_{3} \eta_{i i k} \eta_{j j k}+a_{4} \eta_{i j k} \eta_{i j k}+a_{5} \eta_{i j k} \eta_{k j i} \\
& +b_{1} \xi_{i i j j} \xi_{k k l l}+b_{2} \xi_{i j k k} \xi_{i j l l}+b_{3} \xi_{i i j k} \xi_{j k l l}+b_{4} \xi_{i i j k} \xi_{l l k j}+b_{5} \xi_{i i j k} \xi_{l l j k}+b_{6} \xi_{i j k l} \xi_{i j k l} \\
& +b_{7} \xi_{i j k l} \xi_{j k l i}+c_{1} \varepsilon_{i i} \xi_{j j k k}+c_{2} \varepsilon_{i j} \xi_{i j k k}+c_{3} \varepsilon_{i j} \xi_{k k i j}+b_{0} \varepsilon_{i i j j} .
\end{aligned}
$$

in which $\lambda$ and $\mu$ are the Lame's constants. $\varepsilon_{i j}, \eta_{i j k}$ and $\xi_{i j k l}(i, j, k, l=1,2,3)$ are the components of the classical strain tensor $\varepsilon$, the first strain gradient tensor $\eta$ and the second strain gradient tensor $\xi$

$$
\begin{gathered}
\varepsilon_{i j}=\frac{1}{2}[\nabla \mathbf{u}+\mathbf{u} \nabla]_{i j}=\frac{1}{2}\left(\frac{\partial u_{j}}{\partial x_{i}}+\frac{\partial u_{i}}{\partial x_{j}}\right), \quad \eta_{i j k}=[\nabla \nabla \mathbf{u}]_{i j k}=\frac{\partial^{2} u_{k}}{\partial x_{i} \partial x_{j}} \\
\xi_{i j k l}=[\nabla \nabla \nabla \mathbf{u}]_{i j k l}=\frac{\partial^{3} u_{l}}{\partial x_{i} \partial x_{j} \partial x_{k}} .
\end{gathered}
$$

The conjugate classical stress, the first higher-order stress and the second higher-order stress are defined by differentiating the strain energy density $\bar{u}$ with respect to the corresponding strain components as

$$
\sigma=\frac{\partial \bar{u}}{\partial \varepsilon}, \quad \tau=\frac{\partial \bar{u}}{\partial \eta}, \quad \pi=\frac{\partial \bar{u}}{\partial \xi}
$$

It can be seen that SSG theory formulation enriches the field equations of classical elasticity theory by means of 16 additional higher-order spatial derivative components of relevant state variables. These higher-order terms are accompanied by a set of higher-order material parameters $a_{i}, b_{i}$ and $c_{i}$. These higher-order material parameters can be linked to intrinsic length-scale parameters, on which scale the behavior of underlying material micro-structure can be captured. To be specific, the equation of force equilibrium for the SSG theory-based media in the presence of a body force $\mathbf{f}$ has the following form,

$$
\nabla \cdot(\sigma-\nabla \cdot \tau+\nabla \nabla: \pi)+\mathbf{f}=0
$$

The rule for multiple scalar multiplication of dyads is $\mathbf{a b}: \mathbf{c d}=(\mathbf{a} \cdot \mathbf{b})(\mathbf{c} \cdot \mathbf{d})$. Upon substituting the strain-displacement relations (2) into the stress-strain relations (3) and the resulting expressions for the stresses into the stress-equation of equilibrium Eq. (4), the displacement-equation of equilibrium is given as

$$
(\lambda+2 \mu) D_{11}^{2} D_{12}^{2} \nabla \nabla \cdot \mathbf{u}-\mu D_{21}^{2} D_{22}^{2} \nabla \times \nabla \times \mathbf{u}+\mathbf{f}=0,
$$

where

and

$$
D_{i j}^{2}=1-l_{i j}^{2} \nabla^{2}, \quad i=1,2 ; \quad j=1,2,
$$

$$
\begin{aligned}
& 2(\lambda+2 \mu) l_{1 j}^{2}=\bar{a}-2 \bar{c} \pm\left[(a-2 c)^{2}-4 \bar{b}(\lambda+2 \mu)\right]^{\frac{1}{2}}, \quad j=1,2 ; \\
& 2 \mu l_{2 j}^{2}=\bar{a}^{\prime}-c_{3} \pm\left[\left(\bar{a}-c_{3}\right)^{2}-4 \bar{b}^{\prime} \mu\right]^{\frac{1}{2}}, \quad j=1,2 .
\end{aligned}
$$


And

$$
\begin{aligned}
& \bar{a}=2\left(a_{1}+a_{2}+a_{3}+a_{4}+a_{5}\right) ; \\
& \bar{b}=2\left(b_{1}+b_{2}+b_{3}+b_{4}+b_{5}+b_{6}+b_{7}\right) ; \\
& \bar{c}=c_{1}+c_{2}+c_{3} ; \quad \bar{a}^{\prime}=2\left(a_{3}+a_{4}\right) ; \quad \bar{b}^{\prime}=\left(b_{5}+b_{6}\right) .
\end{aligned}
$$

It can be observed that the displacement equilibrium in Eq. (5) contains terms involving additional material constants $l_{i j}$ having the dimension of length. Therefore $l_{i j}$ are known as intrinsic length scales of SSG theory-based continua. The capability of the SSG theory to capture the size effect is the direct manifestation of involvement of these characteristic lengths [23]. In fact, not only the SSG theory but also all the other types of generalized continuum theory contain such kind of intrinsic length-scale parameters in theory that allows us to model physical phenomena where the micro-structure clearly exhibits specific features with particular length scales. Therefore, the response of a material point not only depends on the strain at that point but also on the strain or history strain of a certain neighborhood of that point or even of the entire body. This is also the reason why generalized continuum theories can be used to capture the complex multi-scale behaviors exhibited by complex media. Certainly, the intrinsic length parameters of the periodic structure and the nano-structure are dependent on their internal structure features, and their length-scale parameters should be chosen in accordance with their intrinsic length.

Length-scale parameters are generally identified by matching the structural properties, including but not limited to: the fundamental frequencies, dispersion curves of plane waves resulting from the generalized theory-based analytical models with those properties calculated by the MD simulations or experiments. For example, in the research of Kandaz [24], the length-scale parameters Modified Strain Gradient (MSG) theory and MCS theory are identified from the existing experimental data of load-displacement curves in literature [25] for the first time. In the same manner, Ansari extracted material length scale parameter by matching the fundamental frequencies obtained from the continuum models with those calculated by the MD simulations [26]. Metrikine et Askes developed a stable and dynamic consistent high-order continuum model with two additional material parameters from a 2D lattice by supposing that the displacement vector of a higher-order continuum should represent an average of the particledisplacements of the underlying lattice [27]. In the research of Domenico [28, 29], length scale identification is carried out by "continualization" a non-local lattice model with both distributed and lumped mass to a three-lengthscale gradient model by employing a higher-order homogenization. In the present work, one direct map operation, that has been used in research [30], is employed to obtain the continuum equation from a lattice, and the long-range interaction parameters are identified in the SSG theory model. We should emphasis that focus of this work is not on the better identification of internal length-scale parameters, more importantly, this paper is focused on the wave conversion and scattering at coupling regions involving non-classical interfaces which are studied based on the SSG theory model. This part is developed in the purpose of interpreting how the length-scale parameters work in governing the global behavior of complex continua and why SSG theory-based model can capture the local behavior by involving higher-order material constants. The higher-order material constants (reflecting instinct length-scale parameters) in the numerical calculation are referred to the results that obtained by atomistic approach [31]. 


\section{Transmission and reflection of a longitudinal wave through a complex rod}

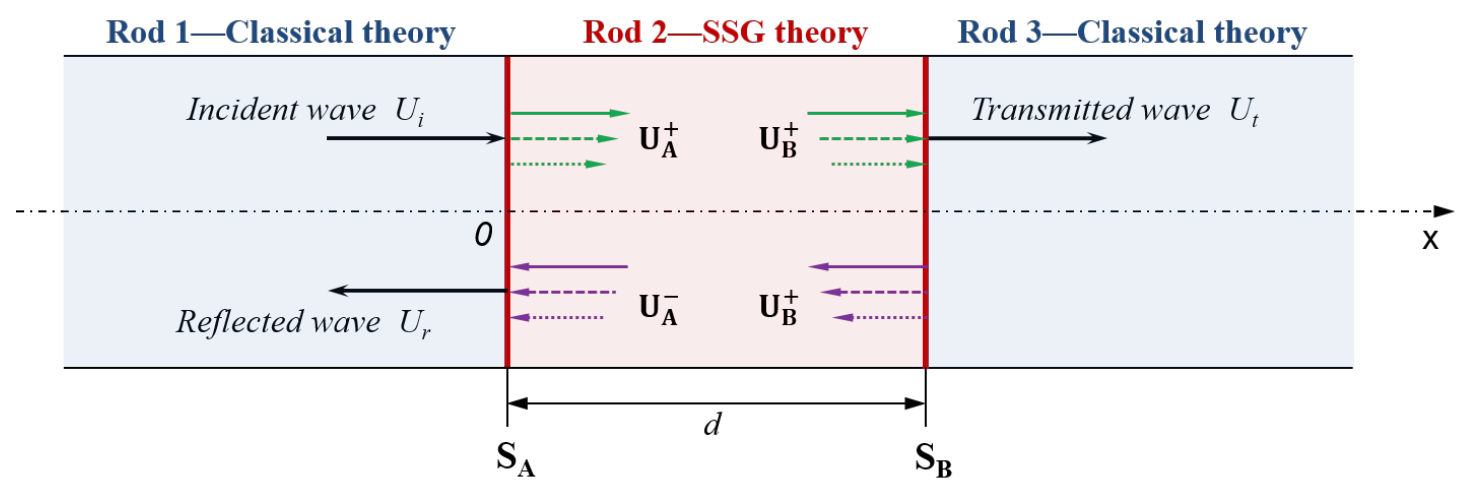

Figure 1: Reflection and transmission of a longitudinal wave through a complex rod of length $d$

Let's consider two classical rods connected by a complex coupling region as show in Figure 1 . Rod 1 and rod 3 are both homogeneous, namely consisting of micro-structures whose size is much smaller than the external wavelength (deformation). Rod 1 and 3 are coupled by Rod 2. As the coupling region, rod 2 is complex, namely the size of its underlying micro-structures is comparable with the external wavelength. Therefore local behavior needs to be captured for a better description of its global vibro-acoustic behavior. The description of local behavior inevitably reveal different features in wave propagation in complex vs. classical media. The most significant is the converted waves in the complex media at wave reflection interfaces. The following analysis is concerned with the wave conversion and scattering at complex coupling regions based on the SSG theory rod model.

\subsection{Wave modes in a complex rod}

Wave propagation in complex rod has been studied by Zhu et al [19] by employing SSG theory-based rod model. Assuming Young's modulus of rod 2 is $E_{2}$, mass density $\rho_{2}$ and circular cross-section area $A=\pi r^{2}$. Along with higher-order material constants are $a_{i}(i=1,2, \ldots 5), b_{i}(i=1,2, \ldots 7)$, and $c_{i}(i=1,2,3)$, strain energy density of the complex rod can be expressed with eq.(1), then the governing equation of the enriched model can be deduced based on Hamilton principle as

$$
E_{2} A \frac{\partial^{2} u}{\partial x^{2}}+\left(B_{3}-B_{1}\right) \frac{\partial^{4} u}{\partial x^{4}}+B_{2} \frac{\partial^{6} u}{\partial x^{6}}+q-m_{0} \frac{\partial^{2} u}{\partial t^{2}}=0,
$$

where $u$ stands for longitudinal displacement in $x$ direction, $m_{0}=\rho_{2} A$ and $B_{i}(i=1,2,3)$ represent the enrichment of higher-order material components as

$$
\begin{aligned}
& B_{1}=2 A\left(a_{1}+a_{2}+a_{3}+a_{4}+a_{5}\right), \\
& B_{2}=2 A\left(b_{1}+b_{2}+b_{3}+b_{4}+b_{5}+b_{6}+b_{7}\right), \\
& B_{3}=2 A\left(c_{1}+c_{2}+c_{3}\right)
\end{aligned}
$$

The associated boundary conditions at the rod ends are given as

$$
\begin{aligned}
& P_{0}=E A \frac{\partial u}{\partial x}+\left(B_{3}-B_{1}\right) \frac{\partial^{3} u}{\partial x^{3}}+B_{2} \frac{\partial^{5} u}{\partial x^{5}} \quad \text { or } \quad \delta u=0, \\
& P_{1}=\left(B_{1}-\frac{B_{3}}{2}\right) \frac{\partial^{2} u}{\partial x^{2}}-B_{2} \frac{\partial^{4} u}{\partial x^{4}} \quad \text { or } \quad \delta\left(\frac{\partial u}{\partial x}\right)=0, \\
& P_{2}=\frac{B_{3}}{2} \frac{\partial u}{\partial x}+B_{2} \frac{\partial^{3} u}{\partial x^{3}} \quad \text { or } \quad \delta\left(\frac{\partial^{2} u}{\partial x^{2}}\right)=0 .
\end{aligned}
$$


Assuming the external loading $q=0$, the dispersion behavior of free wave modes in complex rod can be achieved by substituting the general exponential form of wave propagation solution into the SSG theory-based governing equation as

$$
E_{2} A k^{2}-\left(B_{3}-B_{1}\right) k^{4}+B_{2} k^{6}-m_{0} \omega^{2}=0
$$

According to the form of this dispersion relation, three different modes can be generated in second strain gradient elastic rod can be generated by the disturbance on the discontinuous interface $\mathrm{S}_{\mathrm{A}}$ including the non-classical longitudinal wave $k_{1}$ and two evanescent waves $k_{2}$ and $k_{3}$ (as concluded in study [19]). It is predictable that wave scattering and transmission through complex media should be different from the classical one.

\subsection{Wave conversion and transmission through a complex rod}

As shown in Fig. 1. longitudinal wave $U_{i}$ propagates towards $+x$ direction then normally inject on interface $\mathrm{S}_{\mathrm{A}}$, a proportion of the incident energy is reflected and propagates away towards $-x$ as reflected wave $U_{r}$, and the remaining energy is transmitted to the coupling region rod 2. Based on the previous analysis, 3 wave modes can be generated by the disturbance on the discontinuous interface $\mathrm{S}_{\mathrm{A}}$, so the transmitted energy continues propagating towards $+x$ direction in form of the generated wave group $U_{A}^{+}$. Assuming the length of rod 2 is short thus all generated wave modes can propagate through rod 2 and inject on interface $\mathrm{S}_{\mathrm{B}}$ at $x=d$ as incident wave group $U_{B}^{+}$, part of the incident energy is transmitted to rod 3 in form of transmitted longitudinal wave $U_{t}$, and the other is reflected in form of wave group $U_{B}^{-}$. The reflected wave modes propagate along $-x$ direction until reach interface $\mathrm{S}_{\mathrm{A}}$ and restart a new circular.

To characterize the vibration transmission and reflection features through the coupling rod, the transmission coefficient of the incident wave $U_{i}$ through rod 2 (complex media) to rod 3 and the reflection coefficient of wave $U_{i}$ from rod 2 are derived in this section. Assuming the longitudinal wave that propagates in rod 1 and rod 3 is denoted as $k_{c}$, the incident wave propagating along $+x$ direction with unit amplitude can be expressed as

$$
U_{i}=e^{i\left(\omega t-k_{c} x\right)}
$$

Due to the micro-structure effect, the state description of the interface for rod 2 requires higher-order kinetic and force information as in Eq. (8). To clarify the transfer of motion and force from $S_{A}$ to $S_{B}$, one state vector $\mathbf{X}$ is defined with including all the kinetic parameters and general forces of complex rod based on SSG theory. For interface $S_{A}$, it is

$$
\mathbf{X}_{A^{+}}=\left[u_{A^{+}}, \frac{\partial u_{A^{+}}}{\partial x}, \frac{\partial^{2} u_{A^{+}}}{\partial x^{2}}, P_{0}, P_{1}, P_{2}\right]_{{\mathrm{S}=\mathrm{S}_{\mathrm{A}^{+}}}^{\mathrm{T}}} .
$$

Assuming the amplitudes of the three positive going wave modes $k_{j}(j=1,2,3)$ generated on interface $\mathrm{S}_{\mathrm{A}}$ are $\mathcal{A}_{j}(j=1,2,3)$, meanwhile the amplitudes of the negative going modes $-k_{j}(j=1,2,3)$ incident to $\mathrm{S}_{\mathrm{A}}$ from $-x$ are $\mathcal{B}_{j}$, the displacement on the right hand of the interface $S_{A}$ can be given in form of the wave superposition as (omitting the time component $e^{i \omega t}$ )

$$
u_{A^{+}}=\sum_{j=1}^{3} \mathcal{A}_{j} e^{-i k_{j} x}+\sum_{j=1}^{3} \mathcal{B}_{j} e^{i k_{j} x}=\mathbf{U} \mathbf{D}^{+} \mathbf{A}+\mathbf{U} \mathbf{D}^{-} \mathbf{B}
$$

in which $\mathbf{U}=\left[\begin{array}{lll}1 & 1 & 1\end{array}\right], \mathbf{D}^{+}=\operatorname{diag}\left(e^{-i k_{1} x}, e^{-i k_{2} x}, e^{-i k_{3} x}\right), \mathbf{D}^{-}=\operatorname{diag}\left(e^{i k_{1} x}, e^{i k_{2} x}, e^{i k_{3} x}\right), \mathbf{A}=\left[\begin{array}{lll}\mathcal{A}_{1} & \mathcal{A}_{2} & \mathcal{A}_{3}\end{array}\right]^{\mathrm{T}}$, and $\mathbf{B}=\left[\begin{array}{ccc}\mathcal{B}_{1} & \mathcal{B}_{2} & \mathcal{B}_{3}\end{array}\right]^{\mathrm{T}}$. The first and second derivatives of longitudinal displacement $u_{A^{+}}$are given as

$$
\begin{aligned}
\frac{\partial u_{A^{+}}}{\partial x} & =\mathbf{U} \mathbf{H D}^{+} \mathbf{A}-\mathbf{U} \mathbf{H D}^{-} \mathbf{B}, \\
\frac{\partial^{2} u_{A^{+}}}{\partial x^{2}} & =\mathbf{U} \mathbf{H}^{2} \mathbf{D}^{+} \mathbf{A}+\mathbf{U H}^{2} \mathbf{D}^{-} \mathbf{B},
\end{aligned}
$$


in which diagonal matrix $\mathbf{H}=\operatorname{diag}\left(-i k_{1}, \quad-i k_{2}, \quad-i k_{3}\right)$ consisting of the coefficients in front of each components caused by first derivative in $x$ direction. With boundary force expressed in eq. 8 , , one can express the amplitudes of the axis forces $P_{0}$, higher-order forces $P_{1}$, and $P_{2}$ produced on the right hand of interface $\mathrm{S}_{\mathrm{A}}$ in the form of

$$
\begin{aligned}
& \left.P_{0}\right|_{\mathrm{S}_{\mathrm{A}}}=\mathbf{U} \mathbf{P}_{0} \mathbf{D}^{+} \mathbf{A}-\mathbf{U} \mathbf{P}_{0} \mathbf{D}^{-} \mathbf{B}, \\
& \left.P_{1}\right|_{\mathrm{S}_{\mathrm{A}}}=\mathbf{U} \mathbf{P}_{1} \mathbf{D}^{+} \mathbf{A}+\mathbf{U} \mathbf{P}_{1} \mathbf{D}^{-} \mathbf{B}, \\
& \left.P_{2}\right|_{\mathrm{S}_{\mathrm{A}}}=\mathbf{U} \mathbf{P}_{2} \mathbf{D}^{+} \mathbf{A}-\mathbf{U} \mathbf{P}_{2} \mathbf{D}^{-} \mathbf{B},
\end{aligned}
$$

in which diagonal matrix $\mathbf{P}_{0}=\operatorname{diag}\left(-i E_{2} A k_{j}-i B_{2} k_{j}{ }^{5}+i\left(B_{3}-B_{1}\right) k_{j}^{3}\right), \mathbf{P}_{1}=\operatorname{diag}\left(\left(\frac{B_{3}}{2}-B_{1}\right) k_{j}{ }^{2}-B_{2} k_{j}^{4}\right)$, and $\mathbf{P}_{2}=$ $\operatorname{diag}\left(-\frac{1}{2} i B_{3} k_{j}+i B_{2} k_{j}^{3}\right)(j=1,2,3)$.

From equations (11), (12), (13) and (14), the state vector is reformulated in the following form:

$$
\mathbf{X}_{A^{+}}=\mathbf{M}_{A} \mathbf{U}_{A^{+}}
$$

in which $\mathbf{U}_{A^{+}}=\left[\begin{array}{c}\mathbf{D}^{+} \mathbf{A} \\ \mathbf{D}^{-} \mathbf{B}\end{array}\right]$, and coefficient matrix $\mathbf{M}_{A}$ can be obtained by combining all the above generalized kinetic variables and the force variables as:

$$
\mathbf{M}_{A}=\left[\begin{array}{cc}
\mathbf{U} & \mathbf{U} \\
\mathbf{U} \mathbf{H} & -\mathbf{U} \mathbf{H} \\
\mathbf{U} \mathbf{H}^{2} & \mathbf{U} \mathbf{H}^{2} \\
\mathbf{U} \mathbf{P}_{0} & -\mathbf{U} \mathbf{P}_{0} \\
\mathbf{U} \mathbf{P}_{1} & \mathbf{U} \mathbf{P}_{1} \\
\mathbf{U} \mathbf{P}_{2} & -\mathbf{U} \mathbf{P}_{2}
\end{array}\right]
$$

Similarly, the state vector on the left hand of the studied interface $S_{B}$ can be given as

$$
\mathbf{X}_{B^{-}}=\left[u_{B^{-}}, \frac{\partial u_{B^{-}}}{\partial x}, \frac{\partial^{2} u_{B^{-}}}{\partial x^{2}}, P_{0}, P_{1}, P_{2}\right]_{{\mathrm{S}=\mathrm{S}_{B^{-}}}^{\mathrm{T}}}
$$

Due to the wave propagation distance along $x$ direction, amplitude of each wave mode is changed by $e^{ \pm k_{j} d}$ which indicates the phase difference caused by propagation distance for mode $\pm k_{j}$. The displacement $u_{B^{-}}$can be given in form of the superposition of contribution from all the wave modes as

$$
u_{B^{-}}=\mathbf{U} \operatorname{diag}\left(e^{-i k_{1} d}, e^{-i k_{2} d}, e^{-i k_{3} d}\right) \mathbf{D}^{+} \mathbf{A}+\mathbf{U} \operatorname{diag}\left(e^{i k_{1} d}, e^{i k_{2} d}, e^{i k_{3} d}\right) \mathbf{D}^{-} \mathbf{B}
$$

Substituting this displacement form into Eq. $\left[16\right.$, one can write the state vector $\mathbf{X}_{B^{-}}$as:

$$
\mathbf{X}_{B}^{-}=\mathbf{M}_{A} \operatorname{diag}\left(e^{-i k_{1} d}, e^{-i k_{2} d}, e^{-i k_{3} d}, e^{i k_{1} d}, e^{i k_{2} d}, e^{i k_{3} d}\right) \mathbf{U}_{A^{+}} .
$$

Comparing Eq. [15] and [18, the transfer relation from state vector $\mathbf{X}_{A^{+}}$to state vector of $\mathbf{X}_{B^{-}}$can be formulated as

$$
\mathbf{X}_{B^{-}}=\mathbf{M}_{A} \operatorname{diag}\left(e^{-i k_{1} d}, e^{-i k_{2} d}, e^{-i k_{3} d}, e^{i k_{1} d}, e^{i k_{2} d}, e^{i k_{3} d}\right) \mathbf{M}_{A}^{-1} \mathbf{X}_{A}^{+}=\mathbf{M} \mathbf{X}_{A}^{+},
$$

in which transfer matrix writes

$$
\mathbf{M}=\mathbf{M}_{A} \operatorname{diag}\left(e^{-i k_{1} d}, e^{-i k_{2} d}, e^{-i k_{3} d}, e^{i k_{1} d}, e^{i k_{2} d}, e^{i k_{3} d}\right) \mathbf{M}_{A}{ }^{-1} .
$$

For certain kinds of complex materials and propagation distance $d$, transfer matrix $\mathbf{M}$ can be explicitly determined.

Assuming the transmission coefficient of the incident wave $U_{i}$ through $\operatorname{rod} 2$ (complex media) to rod 3 is $C_{t}$, and the reflection coefficient of wave $U_{i}$ from $\operatorname{rod} 2$ is $C_{r}$, transmitted wave $U_{t}$ in rod 3 and reflected wave $U_{r}$ in $\operatorname{Rod} 1$ can be expressed as

$$
\begin{aligned}
& U_{t}=C_{t} \cdot e^{i\left(\omega t-k_{c} x\right)}, \\
& U_{r}=C_{r} \cdot e^{i\left(\omega t+k_{c} x\right)} .
\end{aligned}
$$


Then the displacements caused by the classical longitudinal wave on the left hand of interface $\mathrm{S}_{\mathrm{A}}$ and on the right hand of interface $\mathrm{S}_{\mathrm{B}}$ can be expressed as (omitting the time component $e^{i \omega t}$ ),

$$
\begin{aligned}
& u_{A^{-}}=e^{-i k_{c} x}+C_{r} \cdot e^{i k_{c} x} ; \\
& u_{B^{+}}=C_{t} \cdot e^{-i k_{c} x} .
\end{aligned}
$$

Subsequently, axis forces produced on the left hand of interface $S_{A}$ and on the right hand of interface $S_{B}$ can be expressed as (omitting the time component $e^{i \omega t}$ ),

$$
\begin{aligned}
& F_{A^{-}}=E_{1} A\left(-i k_{c}\right) \cdot e^{-i k_{c} x}+E_{1} A\left(i k_{c}\right) C_{r} \cdot e^{i k_{c} x} ; \\
& F_{B^{+}}=C_{t} E_{1} A\left(-i k_{c}\right) \cdot e^{-i k_{c} x} .
\end{aligned}
$$

in which $E_{1}$ is Young's modulus of rod 1 and rod 3. Substitute $x=0$ for interface $S_{\mathrm{A}}$ and $x=d$ for interface $S_{\mathrm{B}}$ into the expression of displacement (21) and force [22, the state vectors on $\mathrm{S}_{\mathrm{A}}$ and $\mathrm{S}_{\mathrm{B}}$ are given as

$$
\begin{aligned}
& \mathbf{X}_{A^{-}}=\left[\begin{array}{c}
u_{A^{-}} \\
F_{A^{-}}
\end{array}\right]=\left[\begin{array}{c}
1+C_{r} \\
-i k_{c} E_{1} A+i k_{c} C_{r} E_{1} A
\end{array}\right] ; \\
& \mathbf{X}_{B^{+}}=\left[\begin{array}{c}
u_{B^{+}} \\
F_{B^{+}}
\end{array}\right]=\left[\begin{array}{c}
C_{t} e^{-i k_{c} d} \\
-i k_{c} C_{t} E_{1} A e^{-i k_{c} d}
\end{array}\right] .
\end{aligned}
$$

Assuming the mass between $\mathrm{S}=\mathrm{S}_{\mathrm{A}}{ }^{+}$and $\mathrm{S}=\mathrm{S}_{\mathrm{A}}^{-}$is infinitesimal, equilibrium at interface $\mathrm{S}_{\mathrm{A}}$ can be developed by establishing displacement continuity and axis force balance. Concerning the higher-order displacements (first and second derivatives of classical displacement) and higher-order forces $\left(P_{1}, P_{2}\right)$ in $\mathbf{X}_{A^{+}}, P_{1}$ and $P_{2}$ are set to zero because the long-range interaction is close zero in classical media as its characteristic length is much shorter than the deformation wavelength. Meanwhile, the higher-order kinetic components of the classical media are not necessarily to be zero, thus they are set to be unknown variables $u_{A^{-}}^{\prime}, u^{\prime \prime}{ }_{A^{-}}$. In consequence, the equilibrium describing the continuity of state vector $\mathbf{X}_{A^{-}}$and $\mathbf{X}_{A^{+}}$is expressed as in Eq. (24)

$$
\left.\left[u_{A^{+}}, \frac{\partial u_{A^{+}}}{\partial x}, \frac{\partial^{2} u_{A^{+}}}{\partial x^{2}}, P_{0}, P_{1}, P_{2}\right]^{\mathrm{T}}\right|_{\mathrm{S}=\mathrm{S}_{\mathrm{A}}^{+}}=\left.\left[1+C_{r}, u_{A^{-}}^{\prime}, u^{\prime \prime} A^{-},-i k_{c} E_{1} A+i k_{c} C_{r} E_{1} A, 0,0\right]\right|_{\mathrm{S}=\mathrm{S}_{\mathrm{A}^{-}}} .
$$

The equilibrium condition is the same when it comes to surface $S_{B}$. The higher-order force components of vector $\mathbf{X}_{B^{-}}$are set to zero, and the higher-order kinetic component are set to be unknown variables ${u^{\prime}}_{B^{+}}$and ${u^{\prime \prime}}_{B^{+}}$. Then the continuity of state vector $\mathbf{X}_{B^{-}}$and $\mathbf{X}_{B^{+}}$gives

$$
\left.\left[u_{B^{-}}, \frac{\partial u_{B^{-}}}{\partial x}, \frac{\partial^{2} u_{B^{-}}}{\partial x^{2}}, P_{0}, P_{1}, P_{2}\right]\right|_{\mathrm{S}=\mathrm{S}_{\mathrm{B}}^{-}}=\left.\left[C_{t} e^{-i k_{c} d}, u_{B^{+}}^{\prime}, u^{\prime \prime} B^{+},-i k_{c} C_{t} E_{1} A e^{-i k_{c} d}, 0,0\right]\right|_{\mathrm{S}=\mathrm{S}_{\mathrm{B}}{ }^{+}}
$$

Combining of Eq. (24), 25) and (19), the transformation from $\mathbf{X}_{A^{-}}$to $\mathbf{X}_{B^{+}}$yields:

$$
\mathbf{X}_{B^{+}}=\mathbf{M} \mathbf{X}_{A^{-}}
$$

which rewrites:

$$
\left[\begin{array}{c}
C_{t} e^{-i k_{c} d} \\
u_{B^{+}}^{\prime} \\
u^{\prime \prime} B^{+} \\
-i k_{c} C_{t} E_{1} A e^{-i k_{c} d} \\
0 \\
0
\end{array}\right]=\mathbf{M}\left[\begin{array}{c}
1+C_{r} \\
u_{A^{-}}^{\prime} \\
u^{\prime \prime} A^{-} \\
-i k_{c} E_{1} A+i k_{c} C_{r} E_{1} A \\
0 \\
0
\end{array}\right] \text {, }
$$

and $\mathbf{M}=\mathbf{M}_{A} \operatorname{diag}\left(e^{-i k_{1} d}, e^{-i k_{2} d}, e^{-i k_{3} d}, e^{i k_{1} d}, e^{i k_{2} d}, e^{i k_{3} d}\right) \mathbf{M}_{A}^{-1}$ denotes the transfer matrix as in Eq. (19). Matrix $\mathbf{M}_{A}$ is governed by a group of variables including the wavenumber $k_{j}$, Young's modulus $E_{2}$, higher-order material parameters $B_{i}$ and cross section area $A$, and $\mathbf{M}_{A}$ can be explicitly determined as long as all the material constants and geometrical parameters of complex rod 2 is fully defined, then the transfer matrix can be fully obtained combined with length of the coupling region $d$. Then the six unknown variables including the $C_{t}$ and $C_{r}$ can be achieved with the equilibrium established in Eq. 27). We can see that wave transmission coefficient $C_{t}$ and reflection coefficient $C_{r}$ are significantly affected by the non-local property of the complex rod along with coupling length $d$. 


\section{Transmission and reflection of a bending wave through a complex Timoshenko beam}

The following analysis is distributed to bending wave conversion and scattering at non-classical coupling interface and transmission through a complex coupling region. The studied system is illustrated in Figure 2, Beams 1 and 3 are classical homogeneous beams, the size of their micro-structures is much smaller than the external wavelength. Beam 2 is the coupling region which is described by SSG theory-based Timoshenko beam model. The description of longrange interactions enriches classical continuum theory with local behavior features concerning the wave propagation in complex media and wave diffusion at wave reflection interfaces.

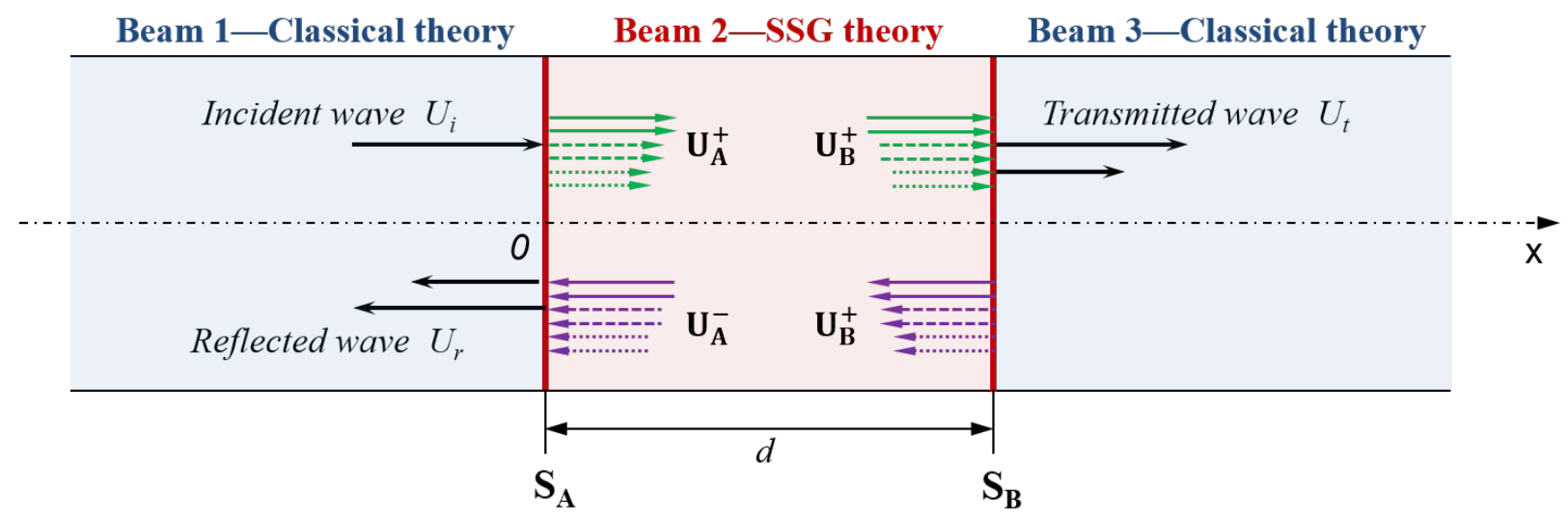

Figure 2: Reflection and transmission of a bending wave through a complex beam of length $d$

\subsection{Wave modes in a complex Timoshenko beam}

Governing equations of the complex Timoshenko beam has been established by Shodja [31] to describe its nonclassical transverse motion $w$ and rotation angle $\psi$. Assuming Young's modulus of beam 2 is $E_{2}$, shear modulus $\mu_{2}$, mass density $\rho_{2}$, along with higher-order material constants $a_{i}(i=1,2, \ldots .5), b_{i}(i=1,2, \ldots 7)$, and $c_{i}(i=1,2,3)$, beam section area $A=b \cdot h$, strain energy density of the complex beam can be expressed, then the governing equation of the complex Timoshenko bean model can be deduced based on Hamilton principle as,

$$
\begin{aligned}
\delta w: & -\mu_{2} A\left(\frac{\partial \psi}{\partial x}\right)-\left(\frac{B_{7}}{2}+\left(c_{3}+c_{2}\right) A\right)\left(\frac{\partial^{3} \psi}{\partial x^{3}}\right)+\frac{B_{8}}{2}\left(\frac{\partial^{5} \psi}{\partial x^{5}}\right)+\mu_{2} A\left(\frac{\partial^{2} w}{\partial x^{2}}\right) \\
& +\left(c_{3} A-B_{5}\right)\left(\frac{\partial^{4} w}{\partial x^{4}}\right)+B_{6}\left(\frac{\partial^{6} w}{\partial x^{6}}\right)+q=m_{0}\left(\frac{\partial^{2} w}{\partial t^{2}}\right) \\
\delta \psi: & -\mu_{2} A \psi+\left(B_{1}-c_{3} A-2 c_{2} A\right)\left(\frac{\partial^{2} \psi}{\partial x^{2}}\right)+\left(B_{4}-B_{2}\right)\left(\frac{\partial^{4} \psi}{\partial x^{4}}\right)+B_{3}\left(\frac{\partial^{6} \psi}{\partial x^{6}}\right) \\
& +\mu_{2} A\left(\frac{\partial w}{\partial x}\right)+\left(\frac{B_{7}}{2}+\left(c_{3}+c_{2}\right) A\right)\left(\frac{\partial^{3} w}{\partial x^{3}}\right)-\frac{B_{8}}{2}\left(\frac{\partial^{5} w}{\partial x^{5}}\right)=m_{2}\left(\frac{\partial^{2} \psi}{\partial t^{2}}\right)
\end{aligned}
$$

in which,

$$
\begin{aligned}
& B_{1}=E_{2} I+2 A\left(a_{1}+2 a_{4}+a_{5}\right), \\
& B_{2}=2 I\left(a_{1}+a_{2}+a_{3}+a_{4}+a_{5}\right)+2 A\left(2 b_{2}+b_{3}+b_{5}+3 b_{6}+2 b_{7}\right), \\
& B_{3}=2 I\left(b_{1}+b_{2}+b_{3}+b_{4}+b_{5}+b_{6}+b_{7}\right), \\
& B_{4}=2 I\left(c_{1}+c_{2}+c_{3}\right), \\
& B_{5}=2 A\left(a_{3}+a_{4}\right), \quad B_{6}=2 A\left(b_{5}+b_{6}\right), \\
& B_{7}=-2 A\left(a_{2}+2 a_{5}\right), \quad B_{8}=-2 A\left(b_{3}+2 b_{4}+2 b_{7}\right),
\end{aligned}
$$


with the term $m_{0}=r h o_{2} A$ as area of beam section and $I=\int_{A} z^{2} d A$ as the area moment of inertia for the beam cross section around $y$ axis. The associated essential and natural boundary conditions at each end (i.e. $x=0, L$ ) are derived as

$$
\begin{aligned}
M_{0}= & \left(B_{1}-A\left(c_{2}+\frac{c_{3}}{2}\right)\right)\left(\frac{\partial \psi}{\partial x}\right)+\left(B_{4}-B_{2}\right)\left(\frac{\partial^{3} \psi}{\partial x^{3}}\right)+B_{3}\left(\frac{\partial^{5} \psi}{\partial x^{5}}\right) \\
& +\left(\frac{B_{7}}{2}+A\left(c_{2}+\frac{c_{3}}{2}\right)\right)\left(\frac{\partial^{2} w}{\partial x^{2}}\right)-\frac{B_{8}}{2}\left(\frac{\partial^{4} w}{\partial x^{4}}\right) \quad \text { or } \delta \psi=0 \\
M_{1}= & A\left(c_{2}+\frac{c_{3}}{2}\right) \psi+\left(B_{2}-\frac{B_{4}}{2}\right)\left(\frac{\partial^{2} \psi}{\partial x^{2}}\right)-B_{3}\left(\frac{\partial^{4} \psi}{\partial x^{4}}\right)-A\left(\frac{c_{3}}{2}+c_{2}\right)\left(\frac{\partial w}{\partial x}\right) \\
& +\frac{B_{8}}{2}\left(\frac{\partial^{3} w}{\partial x^{3}}\right) \quad \text { or } \quad \delta\left(\frac{\partial \psi}{\partial x}\right)=0 \\
M_{2}= & \frac{B_{4}}{2}\left(\frac{\partial \psi}{\partial x}\right)+B_{3}\left(\frac{\partial^{3} \psi}{\partial x^{3}}\right) \quad \text { or } \quad \delta\left(\frac{\partial^{2} \psi}{\partial x^{2}}\right)=0 \\
V_{0}= & -\mu A \psi-\left(\frac{B_{7}}{2}+\left(c_{3}+c_{2}\right) A\right)\left(\frac{\partial^{2} \psi}{\partial x^{2}}\right)+\frac{B_{8}}{2}\left(\frac{\partial^{4} \psi}{\partial x^{4}}\right)+\mu A\left(\frac{\partial w}{\partial x}\right) \\
& +\left(c_{3} A-B_{5}\right)\left(\frac{\partial^{3} w}{\partial x^{3}}\right)+B_{6}\left(\frac{\partial^{5} w}{\partial x^{5}}\right) \quad \text { or } \delta w=0 \\
V_{1}= & \left.\frac{B_{7}+c_{3} A}{2}\right)\left(\frac{\partial \psi}{\partial x}\right)-\frac{B_{8}}{2}\left(\frac{\partial^{3} \psi}{\partial x^{3}}\right)+\left(B_{5}-\frac{c_{3} A}{2}\right)\left(\frac{\partial^{2} w}{\partial x^{2}}\right) \\
& -B_{6}\left(\frac{\partial^{4} w}{\partial x^{4}}\right) \quad \text { or } \delta\left(\frac{\partial w}{\partial x}\right)=0 \\
V_{2}= & -\frac{c_{3} A}{2} \psi+\frac{B_{8}}{2}\left(\frac{\partial^{2} \psi}{\partial x^{2}}\right)+\frac{c_{3} A}{2}\left(\frac{\partial w}{\partial x}\right)+B_{6}\left(\frac{\partial^{3} w}{\partial x^{3}}\right) \quad \text { or } \quad \delta\left(\frac{\partial w}{\partial x}\right)=0
\end{aligned}
$$

Assuming the external loading $q=0$, the dispersion behavior of free wave propagation in enriched Timoshenko beam model can be obtained by substituting the general exponential solution of wave propagation into the governing equation as,

$$
k_{12} k^{12}+k_{10} k^{10}+k_{8} k^{8}+k_{6} k^{6}+k_{4} k^{4}+k_{2} k^{2}+k_{0}=0
$$

in which,

$$
\begin{aligned}
& k_{12}=-B_{3} B_{6} ; \quad k_{10}=\frac{B_{8}^{2}}{4}-B_{3} B_{5}+\left(B_{4}-B_{2}\right) B_{6}+c_{3} A B_{3} ; \\
& k_{8}=B_{4} B_{5}-B_{2} B_{5}-B_{1} B_{6}+\frac{B_{7} B_{8}}{2}+c_{3} A\left(B_{2}-B_{4}+B_{6}+B_{8}\right)+2 c_{2} A B_{6}+c_{2} A B_{8}-\mu_{2} A B_{3} ; \\
& k_{6}=\frac{B_{7}^{2}}{4}+A^{2} c_{2}^{2}-B_{1} B_{5}+c_{2} A\left(2 B_{5}+B_{7}\right)+c_{3} A\left(B_{1}+B_{5}+B_{7}\right) \\
& \quad-A \mu_{2}\left(B_{2}-B_{4}+B_{6}-B_{8}\right)+\omega^{2}\left(B_{3} m_{0}+B_{6} m_{2}\right) ; \\
& k_{4}=m_{0} \omega^{2}\left(B_{2}-B_{4}\right)+m_{2} \omega^{2}\left(B_{5}-c_{3} A\right)-A \mu_{2}\left(B_{5}+B_{7}+B_{1}\right) ; \\
& k_{2}=m_{0} \omega^{2}\left(B_{1}-2 c_{2} A-c_{3} A+\frac{m_{2}}{m_{0}} \mu_{2} A\right) ; \quad k_{0}=m_{0} \omega^{2}\left(A \mu_{2}-m_{2} \omega^{2}\right) .
\end{aligned}
$$

We can see six wave modes can be generated in the second strain gradient elastic beam by a disturbance on discontinuous interface $\mathrm{S}_{\mathrm{A}}$. Let's say they are non-classical bending wave $k_{1}$, non-classical shear wave $k_{2}$ and four evanescent waves $k_{3}, k_{4}, k_{5}$ and $k_{6}$. Wave conversion and transmission through a complex beam is significantly different from the classical one. 


\subsection{Wave conversion and transmission through a complex beam}

Assuming one bending wave $U_{i}$ propagates from negative infinity towards $+x$ direction and normally injects on interface $\mathrm{S}_{\mathrm{A}}$ at $x=0$, a proportion of the incident energy is reflected and propagate away towards $-x$ direction in form of reflected wave group $U_{r}$. The remaining energy is transmitted to the coupling region beam 2 . Based on the previous dispersion relation, 6 wave modes can be generated by the disturbance on the discontinuous interface, therefore the transmitted energy continues propagating to $+x$ direction in forms of generated wave group $U_{A}^{+}$. Assuming the length of the coupling region is short, all generated wave modes on $S_{A}$ can propagate through the beam 2 and inject on interface $\mathrm{S}_{\mathrm{B}}$ at $x=d$ as an incident wave group $U_{B}^{+}$. Then part of the incident energy will be transmitted to beam 3 as transmitted wave group $U_{t}$, and the other is reflected in form of wave group $U_{B}^{-}$. The reflected wave group $U_{B}^{-}$ propagates along $-x$ direction until it reaches interface $\mathrm{S}_{\mathrm{A}}$, possible reflection again generates a steady state.

To characterize the vibration transmission and reflection properties for the complex coupling region, transmission coefficients of the incident bending wave $U_{i}$ through beam 2 (complex media) to beam 3 and the reflection coefficients from beam 2 are derived. Denoting $k_{b}$ and $k_{s}$ respective bending and shear waves propagating in beams 1 and 3 , the incident wave propagating along $+x$ direction with unit amplitude can be expressed as follows:

$$
U_{i}=e^{i\left(\omega t-k_{b} x\right)} .
$$

State description of interface $S_{A}$ in beam 2 should be enriched with higher-order kinetic and force information as in Eq. (8). To clarify the transfer of motion and forces from interface $S_{A}$ to interface $S_{B}$, state vector $\mathbf{X}$ is defined with all the kinetic parameters and force components generated on the interface of the complex beam based on SSG theory. For interface $\mathrm{S}_{\mathrm{A}}$, it is described as:

$$
\mathbf{X}_{A^{+}}=\left.\left[\begin{array}{llllllllllll}
w & \frac{\partial w}{\partial x} & \frac{\partial^{2} w}{\partial x^{2}} & \psi & \frac{\partial \psi}{\partial x} & \frac{\partial^{2} \psi}{\partial x^{2}} & V_{0} & V_{1} & V_{2} & M_{0} & M_{1} & M_{2}
\end{array}\right]^{\mathrm{T}}\right|_{\mathrm{S}=\mathrm{S}_{\mathrm{A}^{+}}} .
$$

Assuming the amplitudes of the positive going wave modes $k_{j}(j=1,2, \ldots 6)$ in wave group $\mathrm{U}_{\mathrm{A}}^{+}$are respectively $\mathcal{A}_{j}$, and the amplitudes of the negative going modes $-k_{j}(j=1,2, \ldots 6)$ in wave group $\mathrm{U}_{\mathrm{A}}^{-}$are $\mathcal{B}_{j}$, then the amplitude of transverse displacement on the right hand of the interface $S_{A}$ can be expressed in wave superposition form (omitting the time component $\left.e^{i \omega t}\right)$ as:

$$
w_{A^{+}}=\sum_{j=1}^{6} \mathcal{A}_{j} e^{-i k_{j} x}+\sum_{j=1}^{6} \mathcal{B}_{j} e^{i k_{j} x}=\mathbf{W}^{+} \mathbf{D}^{+} \mathbf{A}+\mathbf{W}^{+} \mathbf{D}^{-} \mathbf{B}
$$

in which $\mathbf{W}^{+}=\left[\begin{array}{cccccc}1 & 1 & 1 & 1 & 1 & 1\end{array}\right], \mathbf{D}^{+}=\operatorname{diag}\left(e^{-i k_{j} x}\right)$ and $\mathbf{D}^{-}=\operatorname{diag}\left(e^{i k_{j} x}\right)$ are diagonal matrices $(j=1,2, \ldots, 6)$, $\mathbf{A}=\left[\begin{array}{llllll}\mathcal{A}_{1} & \mathcal{A}_{2} & \mathcal{A}_{3} & \mathcal{A}_{4} & \mathcal{A}_{5} & \mathcal{A}_{6}\end{array}\right]^{\mathrm{T}}$, and $\mathbf{B}=\left[\begin{array}{llllll}\mathcal{B}_{1} & \mathcal{B}_{2} & \mathcal{B}_{3} & \mathcal{B}_{4} & \mathcal{B}_{5} & \mathcal{B}_{6}\end{array}\right]^{\mathrm{T}}$. The first and second derivatives of transverse displacement $w_{A^{+}}$can also be deduced as:

$$
\begin{gathered}
\frac{\partial w_{A^{+}}}{\partial x}=\mathbf{W}^{+} \mathbf{H D}^{+} \mathbf{A}-\mathbf{W}^{+} \mathbf{H} \mathbf{D}^{-} \mathbf{B}, \\
\frac{\partial^{2} w_{A^{+}}}{\partial x^{2}}=\mathbf{W}^{+} \mathbf{H}^{2} \mathbf{D}^{+} \mathbf{A}+\mathbf{W}^{+} \mathbf{H}^{2} \mathbf{D}^{-} \mathbf{B},
\end{gathered}
$$

in which diagonal matrix $\mathbf{H}=\operatorname{diag}\left(-i k_{j}\right)(j=1,2, \ldots, 6)$, consisting of the coefficients in front of each mode component caused to express the first derivative in $x$ direction.

Similarly, the amplitude of rotation angle on the right-hand of the interface $S_{A}$ can be expressed as

$$
\psi_{A^{+}}=\mathbf{W}^{+} \boldsymbol{\alpha}^{+} \mathbf{D}^{+} \mathbf{A}-\mathbf{W}^{+} \boldsymbol{\alpha}^{+} \mathbf{D}^{-} \mathbf{B} .
$$

in which $\boldsymbol{\alpha}^{+}$is one diagonal matrix consisting of elements $\alpha_{j}$ as $j t h$ diagonal elements to express the amplitude ratio in eigenvector corresponding to wave mode $+k_{j}$.

$$
\alpha_{j}=\frac{-2 i\left(-B_{6} k_{j}{ }^{6}+c_{3} A k_{j}{ }^{4}-B_{5} k_{j}{ }^{4}-\mu_{2} A k_{j}{ }^{2}+m_{0} \omega^{2}\right)}{k_{j}\left(B_{8} k_{j}{ }^{4}+2 c_{2} A k_{j}{ }^{2}+2 c_{3} A k_{j}{ }^{2}+B_{7} k_{j}{ }^{2}-2 \mu_{2} A\right)} ;(j=1,2 \ldots 6)
$$


Then the first and the second derivatives of rotation angle are written:

$$
\begin{gathered}
\frac{\partial \psi_{A^{+}}}{\partial x}=\mathbf{W}^{+} \mathbf{H} \boldsymbol{\alpha}^{+} \mathbf{D}^{+} \mathbf{A}+\mathbf{W}^{+} \mathbf{H} \boldsymbol{\alpha}^{+} \mathbf{D}^{-} \mathbf{B}, \\
\frac{\partial^{2} \psi_{A^{+}}}{\partial x^{2}}=\mathbf{W}^{+} \mathbf{H}^{2} \boldsymbol{\alpha}^{+} \mathbf{D}^{+} \mathbf{A}-\mathbf{W}^{+} \mathbf{H}^{2} \boldsymbol{\alpha}^{+} \mathbf{D}^{-} \mathbf{B} .
\end{gathered}
$$

With the expressions provided before, amplitudes of the shear forces $V_{0}$ and moment $M_{0}$, higher-order forces $V_{1}, V_{2}$ and moments $M_{1}$ and $M_{2}$ produced on the right interface $\mathrm{S}_{\mathrm{A}}$ are derived and rewritten as follows:

$$
\begin{array}{lll}
\left.M_{0}\right|_{\mathrm{S}_{\mathrm{A}}^{+}}=\mathbf{W}^{+} \mathbf{M}_{0} \mathbf{D}^{+} \mathbf{A}+\mathbf{W}^{+} \mathbf{M}_{0} \mathbf{D}^{-} \mathbf{B}, & \left.V_{0}\right|_{\mathrm{S}_{\mathrm{A}}^{+}}=\mathbf{W}^{+} \mathbf{V}_{0} \mathbf{D}^{+} \mathbf{A}-\mathbf{W}^{+} \mathbf{V}_{0} \mathbf{D}^{-} \mathbf{B}, \\
\left.M_{1}\right|_{\mathrm{S}_{\mathrm{A}}^{+}}=\mathbf{W}^{+} \mathbf{M}_{1} \mathbf{D}^{+} \mathbf{A}-\mathbf{W}^{+} \mathbf{M}_{1} \mathbf{D}^{-} \mathbf{B}, & \left.V_{1}\right|_{\mathrm{S}_{\mathrm{A}}}=\mathbf{W}^{+} \mathbf{V}_{1} \mathbf{D}^{+} \mathbf{A}+\mathbf{W}^{+} \mathbf{V}_{1} \mathbf{D}^{-} \mathbf{B}, \\
\left.M_{2}\right|_{\mathrm{S}_{\mathrm{A}}^{+}}=\mathbf{W}^{+} \mathbf{M}_{2} \mathbf{D}^{+} \mathbf{A}+\mathbf{W}^{+} \mathbf{M}_{2} \mathbf{D}^{-} \mathbf{B}, & \left.V_{2}\right|_{\mathrm{S}_{\mathrm{A}}}=\mathbf{W}^{+} \mathbf{V}_{2} \mathbf{D}^{+} \mathbf{A}-\mathbf{W}^{+} \mathbf{V}_{2} \mathbf{D}^{-} \mathbf{B},
\end{array}
$$

From equations (32)- 38, the state vector at interface $\mathrm{S}_{\mathrm{A}}$ in the complex media 2 can be reformulated as

$$
\mathbf{X}_{A^{+}}=\mathbf{M}_{A} \mathbf{U}_{A^{+}}
$$

in which $\mathbf{U}_{A^{+}}=\left[\begin{array}{c}\mathbf{D}^{+} \mathbf{A} \\ \mathbf{D}^{-} \mathbf{B}\end{array}\right]$, and coefficient matrix $\mathbf{M}_{A}$ can be obtained by combining all the above generalized kinetic variables as well as the force and moment variables as

$$
\mathbf{M}_{A}=\left[\begin{array}{cc}
\mathbf{W}^{+} & \mathbf{W}^{+} \\
\mathbf{W}^{+} \mathbf{H} & -\mathbf{W}^{+} \mathbf{H} \\
\mathbf{W}^{+} \mathbf{H}^{2} & \mathbf{W}^{+} \mathbf{H}^{2} \\
\mathbf{W}^{+} \boldsymbol{\alpha}^{+} & -\mathbf{W}^{+} \boldsymbol{\alpha}^{+} \\
\mathbf{W}^{+} \mathbf{H} \boldsymbol{\alpha}^{+} & \mathbf{W}^{+} \mathbf{H} \boldsymbol{\alpha}^{+} \\
\mathbf{W}^{+} \mathbf{H}^{2} \boldsymbol{\alpha}^{+} & -\mathbf{W}^{+} \mathbf{H}^{2} \boldsymbol{\alpha}^{+} \\
\mathbf{W}^{+} \mathbf{M}_{0} & \mathbf{W}^{+} \mathbf{M}_{0} \\
\mathbf{W}^{+} \mathbf{M}_{1} & -\mathbf{W}^{+} \mathbf{M}_{1} \\
\mathbf{W}^{+} \mathbf{M}_{2} & \mathbf{W}^{+} \mathbf{M}_{2} \\
\mathbf{W}^{+} \mathbf{V}_{0} & -\mathbf{W}^{+} \mathbf{V}_{0} \\
\mathbf{W}^{+} \mathbf{V}_{1} & \mathbf{W}^{+} \mathbf{V}_{1} \\
\mathbf{W}^{+} \mathbf{V}_{2} & -\mathbf{W}^{+} \mathbf{V}_{2}
\end{array}\right]
$$

Due to the wave propagation distance $d$ along $x$ direction, the amplitude of each wave mode is changed by $e^{ \pm k_{j} d}$ indicating the phase difference caused by propagation distance for wave mode $\pm k_{j}$. The state vector on the left hand of interface $\mathrm{S}_{\mathrm{B}}$ writes:

$$
\mathbf{X}_{B^{-}}=\mathbf{M}_{A} \mathbf{D} \mathbf{U}_{A^{+}}
$$

where

$$
\mathbf{D}=\operatorname{diag}\left(e^{-i k_{1} d}, e^{-i k_{2} d}, e^{-i k_{3} d}, e^{-i k_{4} d}, e^{-i k_{5} d}, e^{-i k_{6} d}, e^{i k_{1} d}, e^{i k_{2} d}, e^{i k_{3} d}, e^{i k_{4} d}, e^{i k_{5} d}, e^{i k_{6} d}\right) .
$$

Comparing equations (39) and (40), the transfer relation between state vector $\mathbf{X}_{A^{+}}$for section $\mathrm{S}_{\mathrm{A}}$ and state vector of $\mathbf{X}_{B^{-}}$for section $S_{\mathrm{B}}$ can be formulated as

$$
\mathbf{X}_{B^{-}}=\mathbf{M}_{A} \mathbf{D M}_{A}^{-1} \mathbf{X}_{A}^{+}=\mathbf{M X}_{A}^{+}
$$

in which the transfer matrix is $\mathbf{M}=\mathbf{M}_{A} \mathbf{D} \mathbf{M}_{A}{ }^{-1}$.

Assuming the transmission coefficients of the bending wave, given an incident bending wave mode $U_{i}$ through beam 2 (complex media) to beam 3 , are $t_{b}$ and $t_{s}$, and the reflection coefficients are $r_{b}$ and $r_{s}$, then the amplitudes of transverse displacements and rotation angles on the left hand of interface $S_{A}$ and on the right hand of interface $S_{B}$ can 
be expressed as (omitting the time component $e^{i \omega t}$ ),

$$
\begin{aligned}
& w_{A^{-}}=e^{-i k_{b} x}+r_{b} \cdot e^{i k_{b} x}+r_{s} \cdot e^{i k_{s} x} ; \\
& \psi_{A^{-}}=\alpha_{k_{b}} e^{-i k_{b} x}-\alpha_{k_{b}} r_{b} \cdot e^{i k_{b} x}-\alpha_{k_{s}} r_{s} \cdot e^{i k_{s} x} ; \\
& w_{B^{+}}=t_{b} \cdot e^{-i k_{b} x}+t_{s} \cdot e^{-i k_{s} x} ; \\
& \psi_{B^{+}}=\alpha_{k_{b}} t_{b} \cdot e^{-i k_{b} x}+\alpha_{k_{s}} t_{s} \cdot e^{-i k_{s} x} ;
\end{aligned}
$$

in which

$$
\alpha_{k_{j}}=-\frac{\kappa \mu_{1} A I k_{j}}{E_{1} I k_{j}^{2}-\rho I \omega^{2}+\kappa \mu_{1} A},
$$

$E_{1}, \mu_{1}$ are Young's modulus and shear modulus of beam 2, $\rho_{1}$ is the mass density, and $\kappa$ is the Timoshenko shear coefficient, depends on the geometry. Normally, $\kappa=5 / 6$ for a rectangular section. With the predefined material constants, amplitudes of the shear forces and bending moments produced on the left hand of interface $\mathrm{S}_{\mathrm{A}}$ and on the right hand of interface $S_{B}$ can be calculated as:

$$
\begin{array}{ll}
V_{A^{-}}=E_{1} I \frac{\partial \psi_{A^{-}}}{\partial x} ; & M_{A^{-}}=\mu_{1} A\left(\frac{\partial w_{A^{-}}}{\partial x}-\psi_{A^{-}}\right) ; \\
V_{B^{+}}=E_{1} I \frac{\partial \psi_{B^{+}}}{\partial x} ; & M_{B^{+}}=\mu_{1} A\left(\frac{\partial w_{B^{+}}}{\partial x}-\psi_{B^{+}}\right) .
\end{array}
$$

Substitute $x=0$ for interface $S_{A}$ and $x=d$ for interface $S_{B}$ into the component expressions of state vector, the state vectors on $\mathrm{S}_{\mathrm{A}}$ and $\mathrm{S}_{\mathrm{B}}$ can be reformulated as

$$
\begin{aligned}
& \mathbf{X}_{A^{-}}=\left[\begin{array}{c}
w_{A^{-}} \\
\psi_{A^{-}} \\
V_{A^{-}} \\
M_{A^{-}}
\end{array}\right]=\left[\begin{array}{c}
1+r_{b}+r_{s} \\
\alpha_{k_{b}}-\alpha_{k_{b}} r_{b}-\alpha_{k_{s}} r_{s} \\
-i k_{b} \alpha_{k_{b}} E_{1} I\left(1+r_{b}\right)-i k_{s} \alpha_{k_{s}} r_{s} E_{1} I ; \\
\mu_{1} A\left(r_{b}-1\right)\left(i k_{b}+\alpha_{k_{b}}\right)+\mu_{1} A r_{s}\left(i k_{s}+\alpha_{k_{s}}\right)
\end{array}\right] \\
& \mathbf{X}_{B^{+}}=\left[\begin{array}{c}
w_{B^{+}} \\
\psi_{B^{+}} \\
V_{B^{+}} e^{-i k_{b} d}+t_{s} e^{-i k_{s} d} \\
M_{B^{+}}
\end{array}\right]=\left[\begin{array}{c}
\alpha_{k_{b}} t_{b} e^{-i k_{b} d}+\alpha_{k_{s}} t_{s} e^{-i k_{s} d} \\
-E_{1} I\left(i k_{b} \alpha_{k_{b}} t_{b} e^{-i k_{b} d}+i k_{s} \alpha_{k_{s}} t_{s} e^{-i k_{s} d}\right) \\
-\mu_{1} A\left[t_{b} e^{-i k_{b} d}\left(i k_{b}+\alpha_{k_{b}}\right)+t_{s} e^{-i k_{s} d}\left(i k_{s}+\alpha_{k_{s}}\right)\right]
\end{array}\right] .
\end{aligned}
$$

Assuming the interface $S_{A}, S_{B}$ does not dissipate energy and the mass between the interfaces is infinitesimal, the equality between the state vectors $\mathbf{X}_{A^{-}}$and $\mathbf{X}_{A^{+}}$, then $\mathbf{X}_{B^{-}}$and $\mathbf{X}_{B^{+}}$can be developed. Regarding the higher-order force and moment elements, $M_{1}, M_{2}$ and $V_{1}, V_{2}$ are set to be zero as the long-range interaction is approaching zero in classical media at the interface, meanwhile the higher-order kinetic component of the classical media are set to be unknown variables $w^{\prime} A^{-}, w^{\prime \prime} A^{-}$and $\psi^{\prime} A^{-}, \psi_{A^{-}}^{\prime \prime}$. In consequence, the equilibrium describing the continuity between state vector $\mathbf{X}_{A^{-}}$is expressed as

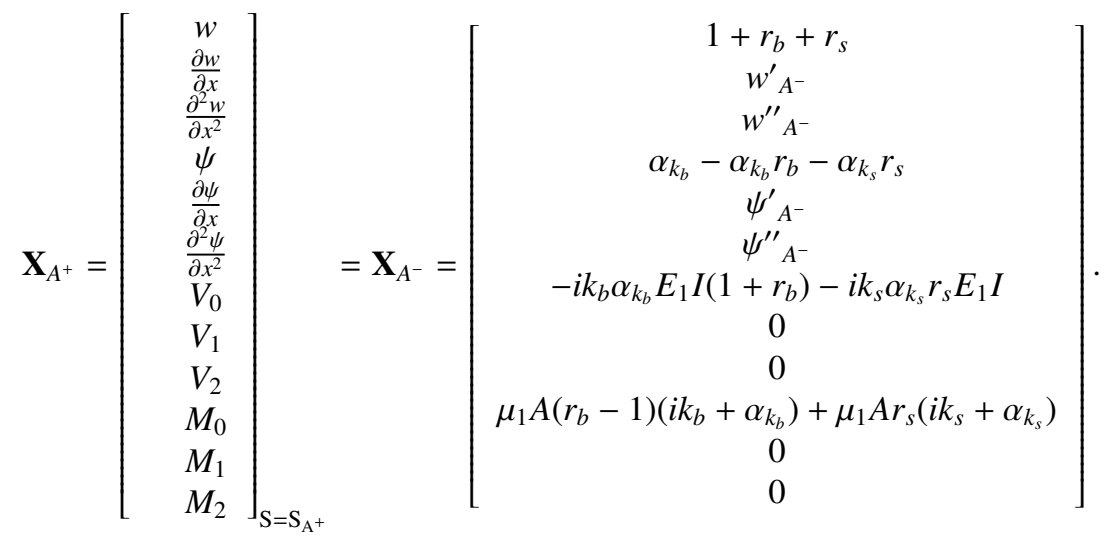


Meanwhile, the equilibrium describing the relation between state vector $\mathbf{X}_{B^{-}}$and $\mathbf{X}_{B^{+}}$is expressed as

$$
\mathbf{X}_{B^{-}}=\left[\begin{array}{c}
w \\
\frac{\partial w}{\partial x} \\
\frac{\partial^{2} w}{\partial x^{2}} \\
\psi \\
\frac{\partial \psi}{\partial x} \\
\frac{\partial^{2} \psi}{\partial x^{2}} \\
V_{0} \\
V_{1} \\
V_{2} \\
M_{0} \\
M_{1} \\
M_{2}
\end{array}\right]_{\mathrm{S}_{=\mathrm{S}^{-}}}=\mathbf{X}_{B^{+}}=\left[\begin{array}{c}
t_{b} e^{-i k_{b} d}+t_{s} e^{-i k_{s} d} \\
w^{\prime} B^{+} \\
w^{\prime \prime} B^{+} \\
\alpha_{k_{b}} t_{b} e^{-i k_{b} d}+\alpha_{k_{s}} t_{s} e^{-i k_{s} d} \\
\psi^{\prime}{ }^{+} \\
\psi^{\prime \prime}{ }_{B^{+}} \\
-E_{1} I\left(i k_{b} \alpha_{k_{b}} t_{b} e^{-i k_{b} d}+i k_{s} \alpha_{k_{s}} t_{s} e^{-i k_{s} d}\right) \\
0 \\
0 \\
-\mu_{1} A\left[t_{b} e^{-i k_{b} d}\left(i k_{b}+\alpha_{k_{b}}\right)+t_{s} e^{-i k_{s} d}\left(i k_{s}+\alpha_{k_{s}}\right)\right. \\
0 \\
0
\end{array}\right]
$$

Combination of Eq.41), 45), and (46) yields:

$$
\mathbf{X}_{B^{+}}=\mathbf{M} \mathbf{X}_{A^{-}}
$$

which rewrites:

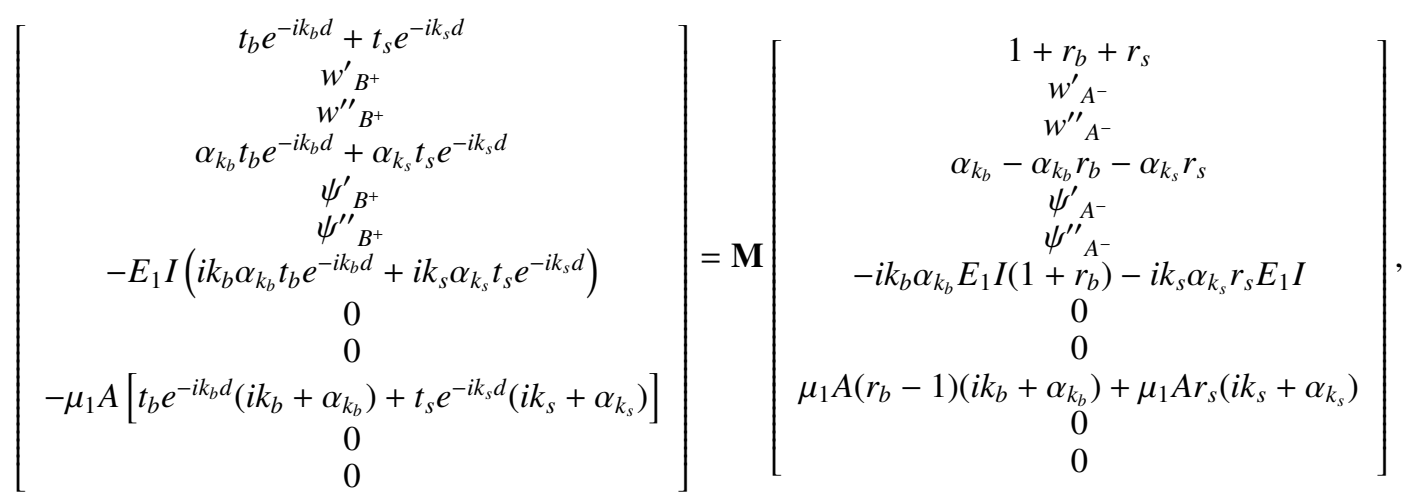

in which $\mathbf{M}$ denotes the same transfer matrix in Eq. (41) with $\mathbf{M}=\mathbf{M}_{A} \mathbf{D} \mathbf{M}_{A}{ }^{-1}$. From the previous formulation, we know that the transfer matrix $\mathbf{M}$ is governed by the not only the classical material properties $\left(E_{2}, m u_{2}, \rho_{2}\right)$ and geometrical parameters $(A, I)$ of beam 2 but also higher-order material parameters $\left(a_{i}, b_{i}, c_{i}\right)$. Along with wave propagation distance $d$, transfer matrix $\mathbf{M}$ can be explicitly determined. Furthermore, we can see that wave transmission and diffusion are significantly affected by the non-local properties of the coupling region.

\section{Numerical calculation of vibration transmission and reflection through a complex region}

\subsection{Method verification}

The SSG theory-based model is characterized by the higher-order material components along with the classical Lame parameters, and it is degenerated into the classical elastic model by setting the higher-order material parameters to zero. Since there is no reference on the results of the SSG theory-based model concerning its scattering properties, the reflection and transmission coefficients through a classical theory-based media can be regarded as the reference result. If the proposed formulation is validated, the wave scattering properties through a non-classical media with very small higher-order material constants tends to degenerate into the classical results. In the numerical verification, wave modes in the CT-based rod model and Timoshenko beam theory are deduced, upon which the wave scattering features of longitudinal wave and bending wave through classical theory-based media are calculated with TMM

Firstly, concerning the formulation of longitudinal wave scattering, one numerical case is calculated for the method verification. Consider the system shown in Figure 1, the radius of the rod section $r=3 a_{0}$ and the length of rod 
Table 1: High-order material constants for aluminum(Shodja et al.,2012).

\begin{tabular}{|c|c|c|c|c|c|c|}
\hline$a_{1}(e V / \stackrel{o}{A})$ & $a_{2}(e V / \stackrel{o}{A})$ & $a_{3}(\mathrm{eV} / \stackrel{\circ}{A})$ & $a_{4}(e V / \stackrel{o}{A})$ & $a_{5}(\mathrm{eV} / \stackrel{\circ}{A})$ & & \\
\hline 0.1407 & 0.0027 & -0.0083 & 0.0966 & 0.2584 & & \\
\hline$b_{1}(\mathrm{eV} \cdot \stackrel{o}{A})$ & $b_{2}(\mathrm{eV} \cdot \stackrel{o}{A})$ & $b_{3}(e V \cdot \stackrel{o}{A})$ & $b_{4}(\mathrm{eV} \cdot \stackrel{o}{A})$ & $b_{5}(\mathrm{eV} \cdot \stackrel{o}{A})$ & $b_{6}(\mathrm{eV} \cdot \stackrel{o}{A})$ & $b_{7}(e V \cdot \stackrel{o}{A})$ \\
\hline $\begin{array}{l}0.7927 \\
c_{1}(e V / \stackrel{o}{A})\end{array}$ & $\begin{array}{l}0.0644 \\
c_{2}(e V / \stackrel{o}{A})\end{array}$ & $\begin{array}{l}-0.1943 \\
c_{3}(e V / \stackrel{o}{A})\end{array}$ & -0.0009 & -0.0009 & 16.1566 & 48.5291 \\
\hline 0.5041 & 0.3569 & 0.1782 & & & & \\
\hline
\end{tabular}

Table 2: High-order material constants for copper(Shodja et al.,2012).

\begin{tabular}{|c|c|c|c|c|c|c|}
\hline$a_{1}(e V / \stackrel{o}{A})$ & $a_{2}(e V / \stackrel{o}{A})$ & $a_{3}(e V / \stackrel{o}{A})$ & $a_{4}(e V / \stackrel{o}{A})$ & $a_{5}(e V / \stackrel{o}{A})$ & & \\
\hline 0.1833 & 0.0103 & 0.0010 & 0.0717 & 0.1891 & & \\
\hline$b_{1}(e V / \stackrel{o}{A})$ & $b_{2}(e V / \stackrel{o}{A})$ & $b_{3}(e V / \stackrel{o}{A})$ & $b_{4}(e V / \stackrel{o}{A})$ & $b_{5}(e V / \stackrel{o}{A})$ & $b_{6}(e V / \stackrel{o}{A})$ & $b_{7}(e V / \stackrel{o}{A})$ \\
\hline $\begin{array}{l}0.6612 \\
c_{1}(e V / \stackrel{o}{A})\end{array}$ & $\begin{array}{l}0.0663 \\
c_{2}(e V / \stackrel{o}{A})\end{array}$ & $\begin{array}{l}-0.2062 \\
c_{3}(e V / \stackrel{o}{A})\end{array}$ & -0.0015 & -0.0015 & 12.6254 & 37.9402 \\
\hline 0.8448 & 0.5732 & 0.3465 & & & & \\
\hline
\end{tabular}

$2 d=4 r$. Material of the rod 1 and $\operatorname{rod} 3$ is aluminum with Young's modulus $E_{1}=52 \mathrm{Gpa}$ and mass density $\rho_{1}=2700 \mathrm{~kg} / \mathrm{m}^{3}$. For the reference CT-based result, the material of rod 2 sandwiched by rod 1 and rod 3 is copper with Young's modulus $E_{2}=80 \mathrm{Gpa}$ and mass density $\rho_{2}=8920 \mathrm{~kg} / \mathrm{m}^{3}$. For the SSG-theory based result, the material of rod 2 is having the same Young's modulus and mass density but also higher-order material constants which are assigned to be only one percent of the experimental values as in Table 2 .

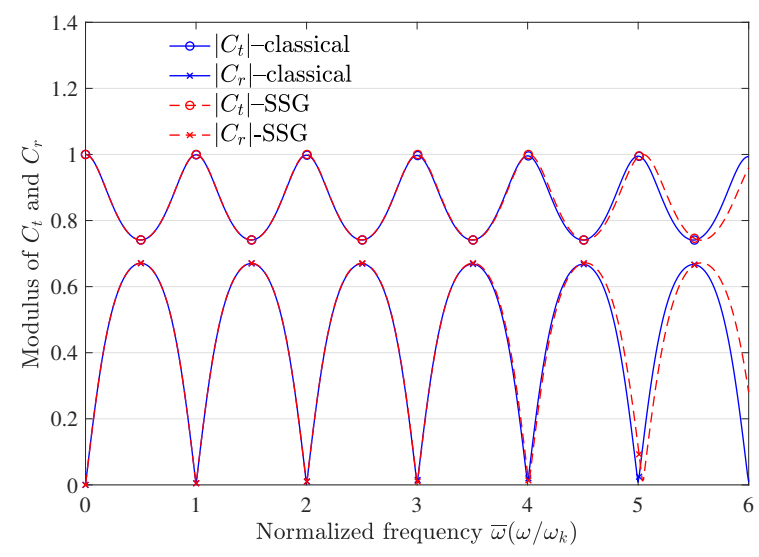

Figure 3: Transmission and reflection coefficients of a longitudinal wave through the classical media (solid lines), and non-classical media with small higher-order material constants (dashed lines)

A good agreement between the SSG theory result and the classical result is seen in Figure 3. When the non-local effects are small, the convergence of the proposed SSG theory-based formulation to the classical result is verified.

Concerning the formulation verification of bending wave scattering, another numerical case is implemented. For the system shown in Figure 2 the height of the rectangular interface $h=5 a_{0}$ and width $b=h$, the normalization parameter for length is set to be the length $d$ of beam 2. Material of beam 1 and beam 3 is aluminum with shear Modulus $\mu=26 \mathrm{Gpa}$. For the reference CT-based result, the material of beam 2 is copper with shear modulus $\mu_{2}=40 \mathrm{Gpa}$ and mass density $\rho_{2}=8920 \mathrm{~kg} / \mathrm{m}^{3}$. For the SSG-based result, the material of beam 2 is having the same 
shear modulus and mass density but also higher-order material constants which are in the value of only one percent of the experimental values as in Table 2 .

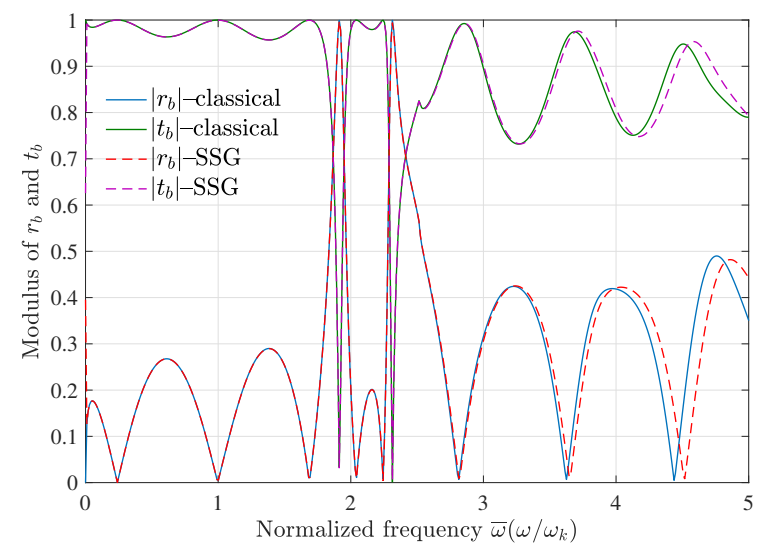

Figure 4: Transmission and reflection coefficients of the bending wave through the classical media (solid lines), and non-classical media with small higher-order material constants (dashed lines)

When the higher-order material constants are very small, non-local effects described by the SSG theory-based model can be neglected, therefor we can see a good agreement between the SSG theory result and the classical result in Figure 3, The convergence from the SSG theory-based formulation to the classical result can be verified. To be noted, the normalization parameter for frequency $\omega_{k}$ is the second order natural frequency of beam 2 calculated by classical Timoshenko beam model, and the non-smooth shape around $\bar{\omega}=2$ is caused by the cut-on frequency of the shear wave.

Meanwhile, although there is very seldom literature contributing to the wave scattering through the non-classical media, we can still find some interesting results to compare with, for example, the reflection and transmission coefficients through a couple-stress elastic slab changing with the incident wave angle and the dimensional thickness in [20], the reflection of elastic waves in the functionally graded piezoelectric microstructures based on modified CS theory [21]. Some of these results can be used to compared qualitatively, for example in Figure 6 [21] when the incident wave angle is 0 the results are in a similar form with the results in section 5.1, and the feature that reflection and transmission coefficients are affected by the dimensionless thickness can also be confirmed in later numerical results.

\subsection{Longitudinal wave transmission and reflection through a complex rod}

In the numerical study, reflection coefficient $C_{r}$ and transmission coefficient $C_{t}$ through the non-local coupling region are calculated for the system shown in Figure 1 . The radius of the section interface $r=3 a_{0}$, and the normalization parameter for length is the length of the non-classical rod $d$. Material of the classical rod 1 and rod 3 is aluminum. Rod 2 is of complex media with its higher-order material constants illustrated in table 1 and table 2 . In the following numerical cases, the coupling region is set to have different materials and different lengths, then the influences of non-local material properties and coupling region length on the $C_{r}$ and $C_{t}$ can be evaluated.

First of all, if rod 2 is homogeneous and its material is the same as rod 1, the incident wave will be fully transmitted without any reflection, in this case, the transmission coefficient is 1 and the reflection coefficient is 0 in the whole frequency range. If the coupling region is complex media and its material is also set to be aluminum, namely having the same Young's modulus and mass density as rod 1 but with higher-order constants, the wave transmission situation will be different. The resulting $C_{t}$ and $C_{r}$ are illustrated in Figure 5 


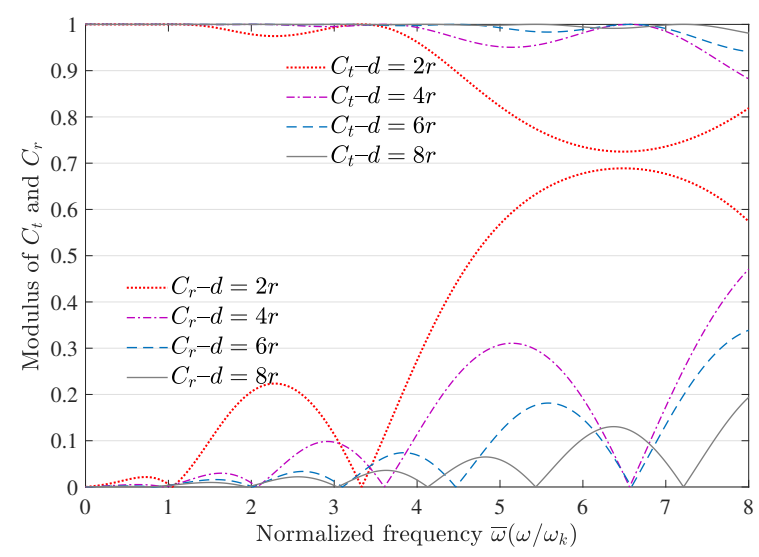

Figure 5: Transmission and reflection coefficients of a longitudinal wave through a complex rod made by Al

We can see $C_{t}$ is not constantly equal to 1 anymore. In fact, the non-classical $C_{t}$ is less than ' 1 ' while $C_{r}$ is far from ' 0 ', and they both display frequency-dependent character. The frequency-dependency reflects that influence of local behavior caused by long-range interaction can be captured by the proposed model and the influence of local behavior differs as external deformation wavelength changes. On some specific frequencies, $C_{t}$ regresses to 1 and $C_{r}$ to 0 as the phase change by wave propagation distance $d$ is approaching to "0" or $\pi$ by coincidence. In this numerical case, rod 2 can be regarded as one kind of architectured media with Young's modulus and mass density the same as the aluminum, and the non-local behavior of this media is significant under the studied frequency band. Meanwhile, the non-local behavior of rod 1 and rod 3 is rather inconspicuous, then the impedance mismatch arises on the coupling interface of these two media from the non-local behaviors description. As the complex coupling length $d$ increases from $2 r$ to $8 r$, the frequency gap between neighboring specific frequencies narrows down and the amplitude of $C_{r}$ becomes lower, the result tends to regress to classical theory results. When the length of rod 2 is increased, the structure is under a relatively longer deformation wavelength, the effect of non-local behavior caused by the underlying micro-structure interaction becomes weak, and the resulting impedance mismatch becomes less obvious and eventually disappears in global behavior description under a relatively longer deformation wavelength. Therefore, $C_{t}$ and $C_{r}$ are both degenerating to classical theory results, which are $C_{t}=1$ and $C_{r}=0$.

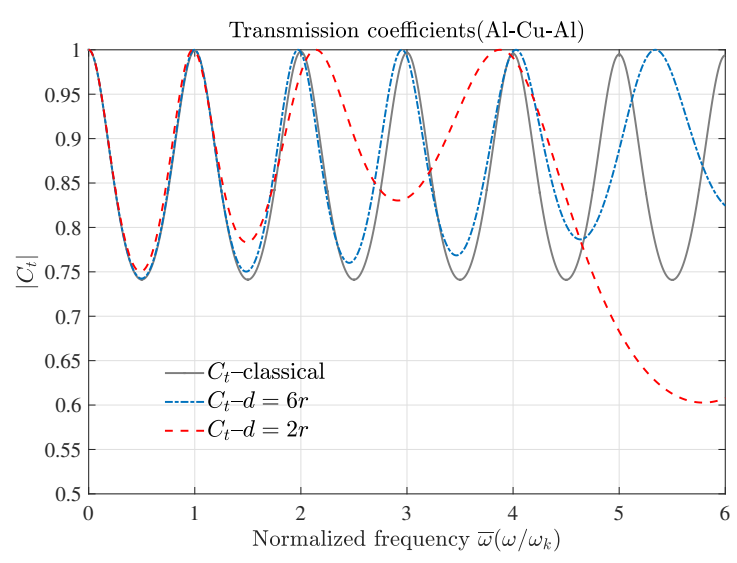

(a)

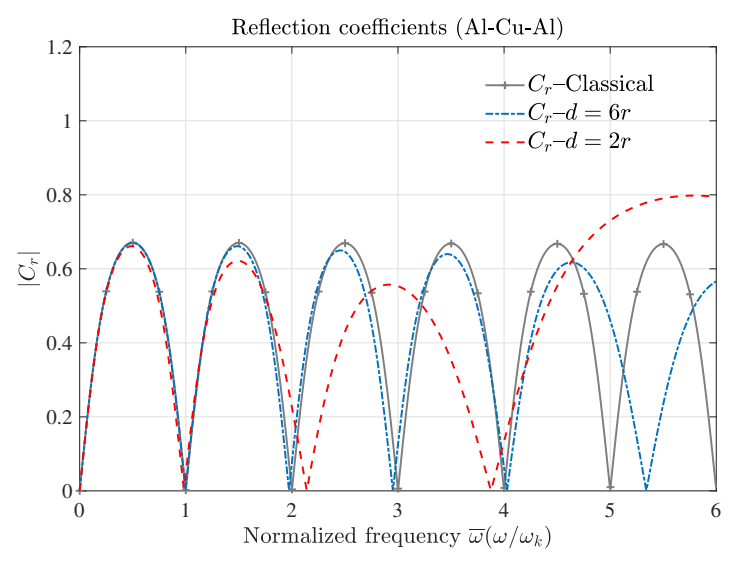

(b)

Figure 6: Transmission and reflection coefficients of a longitudinal wave through a complex rod made by $\mathrm{Cu}$

Figure 6illustrates transmission coefficient $C_{t}$ and reflection coefficient $C_{r}$ of a longitudinal wave through a complex coupling region made of copper. As can be seen, $C_{t}$ and $C_{r}$ resulting from SSG theory formulation match well 
with classical theory result in the low-frequency range, but their difference becomes more distinct as the frequency goes up, namely, the deformation wavelength becomes short and comparable to the characteristic length of internal heterogeneity. Meanwhile, as length $d$ increases from $2 r$ to $6 r, C_{t}$ and $C_{r}$ is observed approaching to classical theory result. The non-local effects on scattering proprieties of the longitudinal wave become more significant in higher frequency and for a short coupling region. To be noted, the classical results, namely the final form that SSG theory model is degenerated to in frequency domain, is different from the case shown in Figure 5 that's because the impedance mismatch comes from not only the higher-order enrichment introduced by the SSG theory model but also different classical material parameters such as Young's modulus and mass density. Both cases are degenerating to classical theory result, but the impedance mismatch in this case will not disappear.

To better illustrate the regressing process of non-classical wave scattering properties to the classical theory results, the transmitted power ratio in form of $\left|C_{t}\right|^{2}$ and reflected power ratio $\left|C_{t}\right|^{2}$ varying with the dimensionless length of the coupling region $d / r$ are plotted as in Figure 7

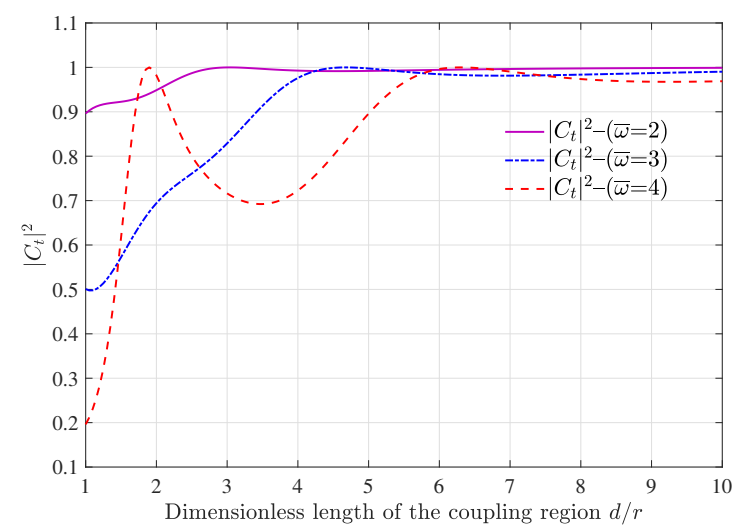

(a)

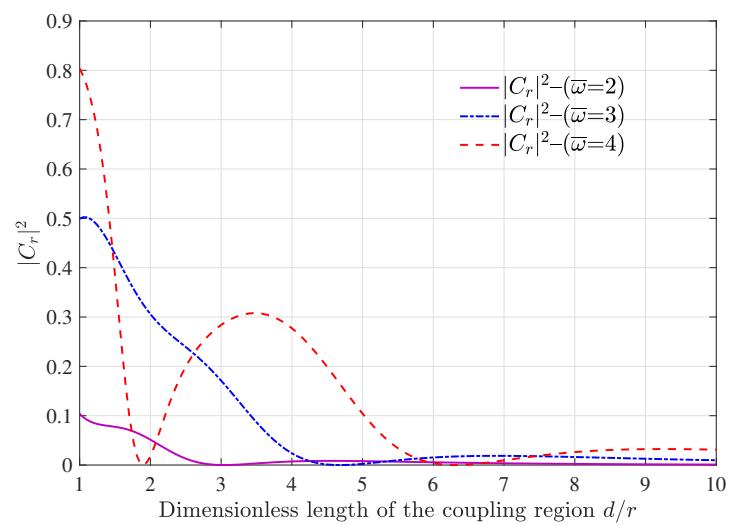

(b)

Figure 7: Transmission and reflection coefficients of a longitudinal wave through a complex region with its dimensionless length

The dimension-dependent characteristic of the transmitted power implies that the impact of local behavior caused by long-range interaction varies with the dimension of the structure. Meanwhile, on three different frequencies, as the length of the coupling region increase, the transmitted power ratios are approaching "1" and the reflected power ratios are close to "0". This phenomenon can also be interpreted combined with Figure 3 As the resulting classical transmission coefficients are all " 1 " and reflection coefficient are all " 0 " on these three specific frequencies $\bar{\omega}=2,3,4$, the changing trend of transmitted and reflected power ratios of the enriched model is theoretically consistent with the degenerated form that it is expected to be.

\subsection{Control of vibration transmission through a complex rod}

From the previous two cases, we can conclude that longitudinal wave transmission through a complex coupling region is different from a classical one. Wave scattering is indeed affected by the local behavior of the complex media, and the influence also varies with the coupling length $d$. However, what is the underlying physical interpretation of this phenomenon? and how can we exploit some applications on this basis? To answer these questions, one lattice model which can physically describe the long-range interactions in the SSG theory-based model is required.

Non-local continuum theory is based on the assumption that the forces between material points are long-range, thus the lattice model for the SSG theory should be capable of reflecting the long-range nature of inter-atomic forces. In the following, a new lattice model is developed to have a discrete micro-structural basis for the SSG theory-based continuum model.

For the studied 1D SSG theory-based rod, the lattice equation of motion is proposed with coupling particles 
describing the long-range interactions as

$$
M \frac{\partial^{2} u_{n}(t)}{\partial t^{2}}=g_{2}\left(u_{n+1}-2 u_{n}+u_{n-1}\right)+g_{4}\left(u_{n+2}-2 u_{n}+u_{n-2}\right)+g_{6}\left(u_{n+3}-2 u_{n}+u_{n-3}\right)+F(n)
$$

where $u_{n}(t)$ are the displacements from the equilibrium. $g_{2}, g_{4}$ and $g_{6}$ represent coupling constants for the three different types of interaction: the nearest-neighbor, the next-nearest-neighbor and the next-next nearest-neighbor interactions. $F_{n}$ indicates the external force applied on the particles. One discrete mass-spring system corresponding to the suggested lattice model is given in Figure 8.

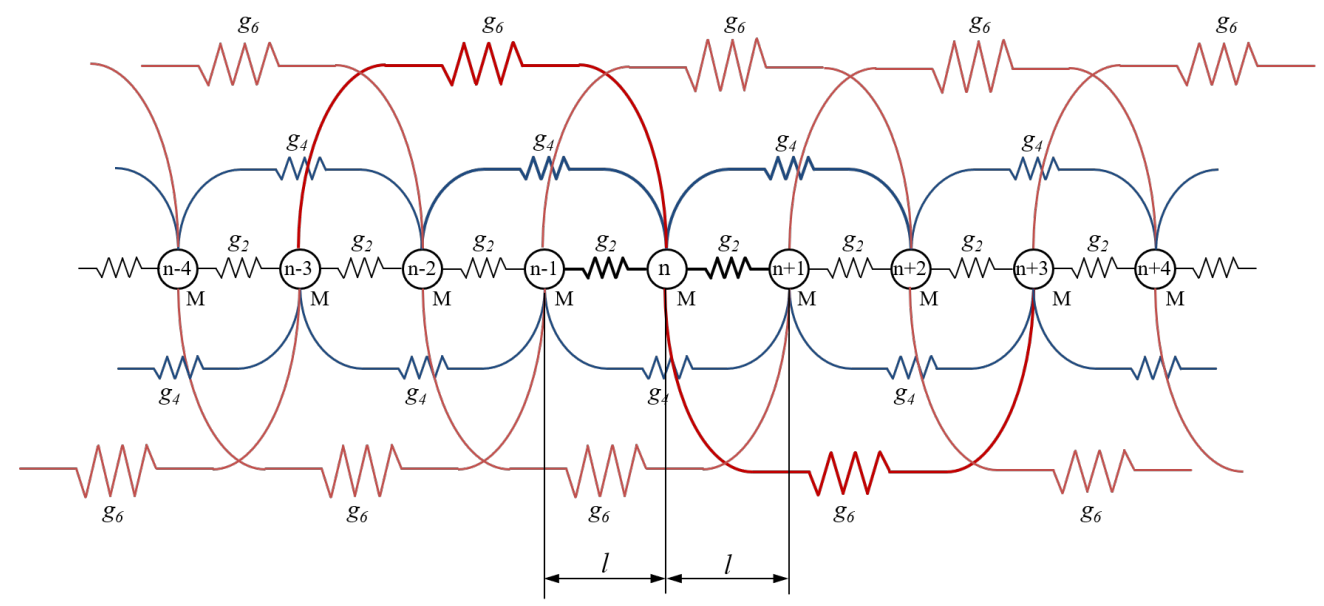

Figure 8: lattice model with nearest-neighbor and next-nearest-neighbor and next-next-nearest-neighbor interactions for SSG theory-based rod

In the lattice model, all the particles have the same mass $\mathrm{M}$, and the infinite lattice has a cross-section area A, and the inter-particle distance $l$. The three types of interactions between particles are separately modeled by springs with stiffness coefficients $k_{2}=g_{2}, k_{4}=g_{4}$ and $k_{6}=g_{6}$.

To achieve a continuum equation from the suggested lattice model, a series is utilized that transforms the equations of motion for $u_{n}(t)$ of lattice model into continuum equation for $u(x, t)$ that describes one-dimensional displacement field [30]. The map operation is introduced in detail in Appendix 1. In the end, the continuum equation can be achieved as

$$
\frac{\partial^{2} u(x, t)}{\partial t^{2}}=G_{2} \frac{\partial^{2} u(x, t)}{\partial x^{2}}+G_{4} \frac{\partial^{4} u(x, t)}{\partial x^{4}}+G_{6} \frac{\partial^{6} u(x, t)}{\partial x^{6}}+F(x)
$$

where

$$
G_{2}=\frac{\left(g_{2}+4 g_{4}+9 g_{6}\right) l^{2}}{M}, G_{4}=\frac{\left(g_{2}+16 g_{4}+81 g_{6}\right) l^{4}}{12 M}, G_{6}=\frac{\left(g_{2}+64 g_{4}+729 g_{6}\right) l^{6}}{360 M}
$$

The 1-D continuous model based on SSG theory has been established based on Hamilton principle in previous work as,

$$
\frac{\partial^{2} u(x, t)}{\partial t^{2}}=\frac{E_{2}}{\rho_{2}} \frac{\partial^{2} u(x, t)}{\partial x^{2}}+\frac{B_{3}-B_{1}}{\rho_{2} A} \frac{\partial^{4} u(x, t)}{\partial x^{4}}+\frac{B_{2}}{\rho_{2} A} \frac{\partial^{6} u(x, t)}{\partial x^{6}}+F(x),
$$

where $\rho_{2}=\frac{M}{A l}$ denotes mass density. Identifying the coefficients from both equations, we obtain the following 
identities:

$$
\begin{aligned}
& G_{2}=\frac{\left(g_{2}+4 g_{4}+9 g_{6}\right) l^{2}}{M}=\frac{E_{2}}{\rho_{2}}, \\
& G_{4}=\frac{\left(g_{2}+16 g_{4}+81 g_{6}\right) l^{4}}{12 M}=\frac{B_{3}-B_{1}}{\rho_{2} A}, \\
& G_{6}=\frac{\left(g_{2}+64 g_{4}+729 g_{6}\right) l^{6}}{360 M}=\frac{B_{2}}{\rho_{2} A} .
\end{aligned}
$$

The stiffness coefficients of the three types of interactions between particles are connected with the material's classical and higher-order parameters as:

$$
\begin{aligned}
& g_{2}=\frac{3 E_{2} A l^{4}+13\left(B_{1}-B_{3}\right) l^{2}+30 B_{2}}{2 l^{5}} \\
& g_{4}=-\frac{3 E_{2} A l^{4}+40\left(B_{1}-B_{3}\right) l^{2}+120 B_{2}}{20 l^{5}} \\
& g_{6}=\frac{E_{2} A l^{4}+15\left(B_{1}-B_{3}\right) l^{2}+90 B_{2}}{90 l^{5}}
\end{aligned}
$$

we can see that the proposed 1D lattice model can be transformed into SSG theory-based continuous model. Young's modulus $E_{2}$ and higher-order material parameters $B_{1}, B_{2}, B_{3}$ are directly connected with the stiffness of general force interactions (short-range and long-range ) along with the inner-particle length $l$ and cross section's area $A$. Therefore the local behavior caused by the complex long-range interactions of internal structures can be captured by the SSG theory-based model. In other words, $g_{2}, g_{4}$ and $g_{6}$, these three type coupling interactions together with inner-particle length $l$ and cross section's area $A$ determine the material parameters $\left(E_{2}, B_{1}, B_{2}, B_{3}\right)$ in SSG theory-based rod model.

The higher-order material constants used in the presented work in table 1 and 2 refer to the research by Shodja et al. (2012) [31]. As interpreted above, higher-order material constants are related to long-range interaction and inner-particle length $l$. In Shodja's research, the crystal lattice of the considered metal is periodically distributed in a nano-scale. Therefore these higher-order material constants, calculated with an atomistic approach, can reflect properties of complex media that have internal structures periodically distributed at nano-scale. Analogically, for any kind of complex media consisting of periodic cells or internal structures, the long-range interaction between non-adjacent internal structures will play an important role in the global structural behavior when the deformation wavelength is comparable to the internal length of micro-structures. The SSG theory-based model as well as other generalized continuum elasticity theories also apply in this case. The higher-order material constants should be determined corresponding to the long-range interaction stiffness. These results indicate that wave transmission through a complex rod can be controlled by adjusting the higher-order material constants, which corresponds to the internal stiffness. In the following, numerical cases are implemented to study the influence of higher-order material constants on wave transmission, with the purpose of obtaining the minimum possible energy transmission.

Figure 9 shows the transmission and reflection coefficients evolving with material constants respectively when $d=$ $r$ and $d=0.5 r\left(E_{1}, \rho_{1}, B_{i}\right.$ are the material parameters for aluminum with $B_{i}$ being calculated with atomistic approach in [31]). As we can see, for complex rod 2 of different length $d$, with some predefined Young's modulus, mass density, and higher-order material parameters, reflection coefficient $C_{r}$ can highly approach ' 1 ' in some frequency ranges. In this frequency range, all of the input energy is reflected and the transmitted energy is close to zero. Thus the predefined complex rod would show great isolation performance in a potential seismic application. This phenomenon could also be used for enriching the inner micro-structure with the utilization of the SSG theory model to achieve better structural performance in acoustics. 


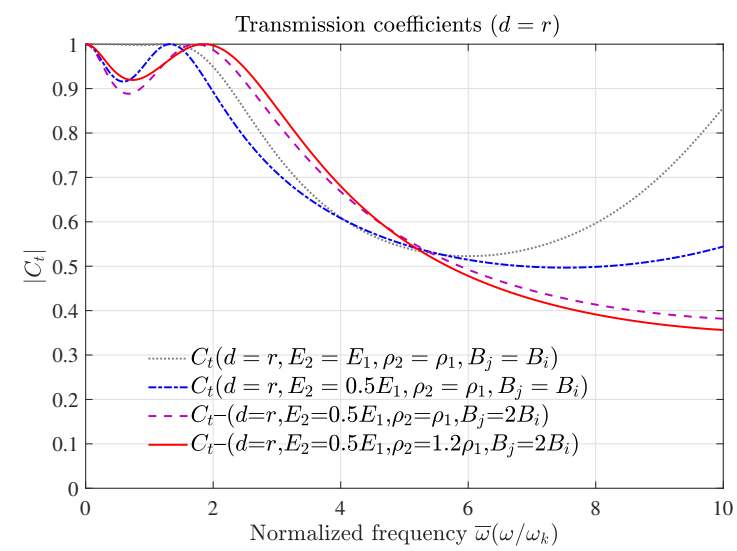

(a)

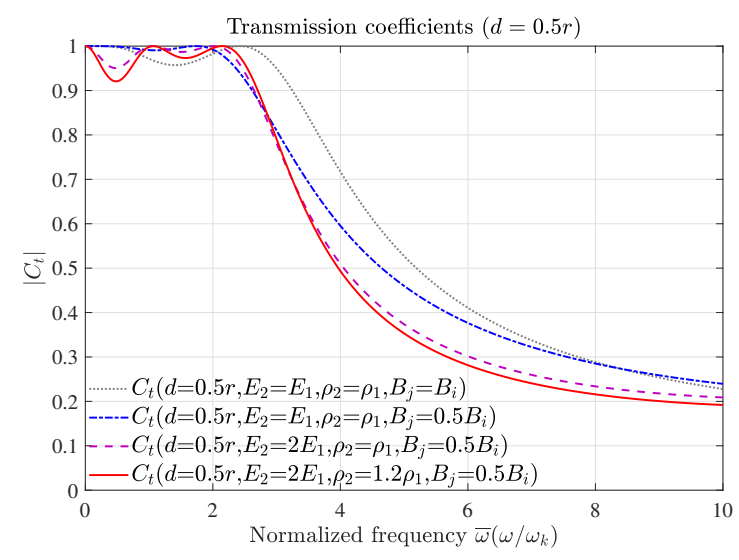

(c)

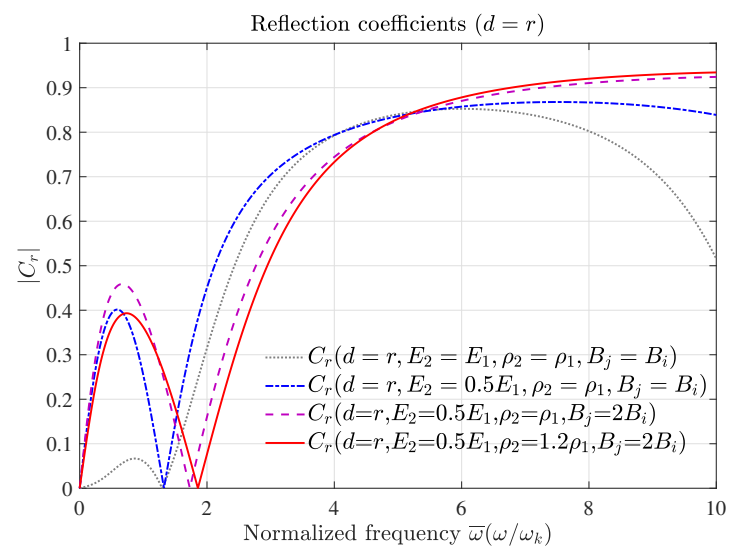

(b)

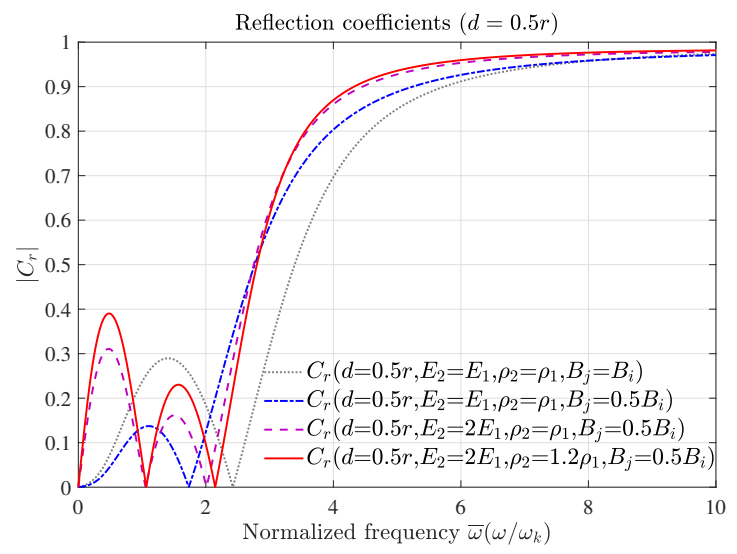

(d)

Figure 9: Transmission and reflection coefficients of a longitudinal wave through a complex rod of different length

Based on these two cases, We can conclude that the vibration transmission and reflection property of the complex coupling region is affected by its length $d$ and all the material constants in the SSG theory model (including the $E_{2}$ and $\rho_{2}$ and higher-order material parameters $B_{i}$ ). Combined with the lattice model proposed above, the force interaction coefficient $g_{2}$ and the long-range force interaction coefficients $g_{4}, g_{6}$ can be also determined by these material constants. Therefore the long-range interaction terms represent the underlying reason that local behaviors caused by inner heterogeneous structure arise. We can say that the structural-acoustic behavior can be designed by adjusting the force interactions between inner structures, which can also be considered as adjusting the material constants as these two groups of variables are equivalence under some conditions. Analogously, wave transmission characteristics through any complex system with internal microstructure can be controlled by adjusting its equivalent force interactions between inner structures.

\subsection{Bending wave transmission and reflection through a complex beam}

In the numerical study, the transmission coefficients $t_{b}$ and $t_{s}$ and reflection coefficients $r_{b}$ and $r_{s}$ are calculated for the system shown in Figure 2. Assuming the height of the rectangular interface $h=5 a_{0}$ and width $b=h$, the normalization parameter for length is set to be the length $d$ of beam 2. The material of the classical media beam 1 and beam 3 is aluminum. In the following analysis, reflection and transmission coefficients through the coupling region are calculated in cases when beam 2 are of different material and different length.

Firstly, if beam 2 is homogeneous and its material is the same as beam 1, the incident bending wave will be transmitted through beam 2 without any reflection nor diffusion. The transmission coefficient of bending wave $t_{b}=1$ and reflection coefficient $r_{b}=0$, while the reflection and transmission coefficients of the converted shear wave $t_{s}=0$ 
and $r_{s}=0$ in the whole frequency range. If beam 2 is complex media, and its material is also aluminum, namely having the same shear modulus and mass density as beam 1 but with higher-order material constants, wave scattering behavior through beam 2 will be different. The resulting reflection coefficients and transmission coefficients of the bending wave are illustrated in Figure 10

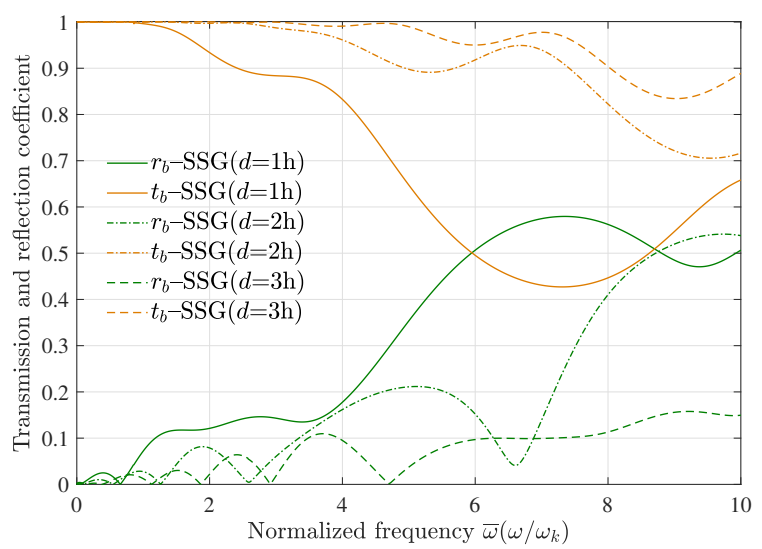

Figure 10: Reflection and transmission coefficients of bending wave and shear wave through a complex beam made of Al

As shown in Figure 10, $t_{b}$ and $r_{b}$ are not constant anymore, instead, these two coefficients are both frequencydependent. The frequency-dependency indicates that the influence of local behavior in the complex coupling region can be captured by the proposed higher gradient-based wave-scattering formulation, and the local behavior becomes more significant under a short wavelength. At certain frequencies, $r_{b}$ is close to 0 and $t_{b}$ to 1 . On these specific frequencies, energy transmitted in beam 2 is totally reflected back by the interface $\mathrm{S}_{\mathrm{B}}$. As $d$ increases from $h$ to $3 h$, the frequency gap between neighboring specific frequencies shows differences, and the amplitude of $r_{b}$ also becomes lower. These phenomena are caused not only by the longer coupling distances, but also the different wave propagation behavior in the coupling region. As the length $d$ increased from $h$ to $3 h$, beam 2 is under a longer dimensionless deformation wavelength, then the long-range interaction becomes less important in global beam behavior description. The impedance mismatch caused by the non-local effect becomes negelectalbe, therefore non-classical bending wave $k_{1}$ tends to approach $r_{b}=0$, and $r_{b}$ is returning to classical theory result zero. 


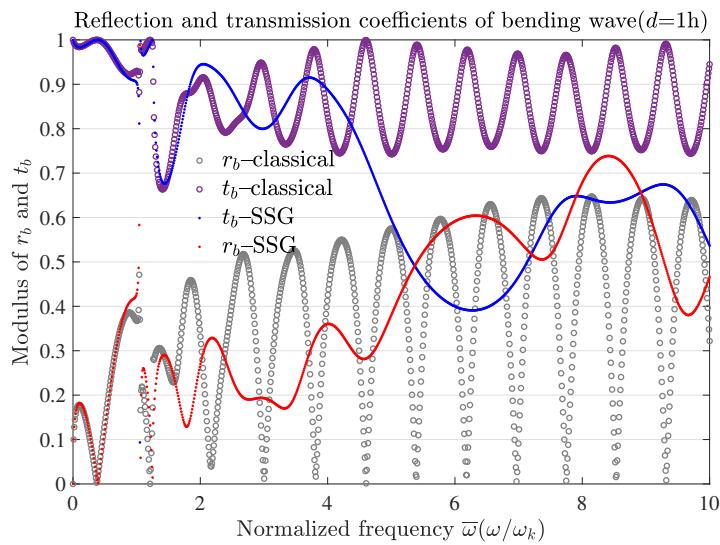

(a)

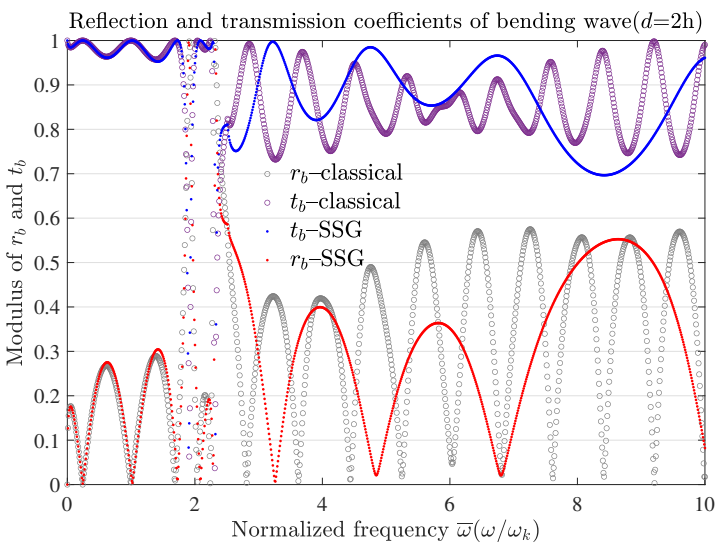

(c)

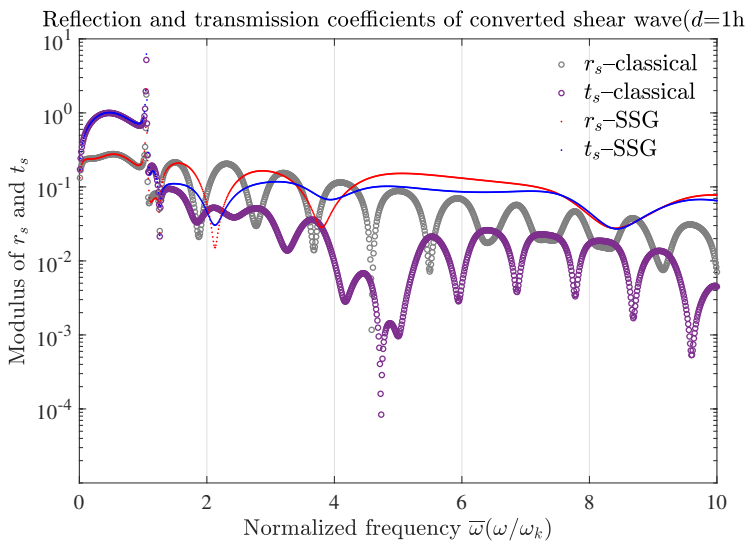

(b)

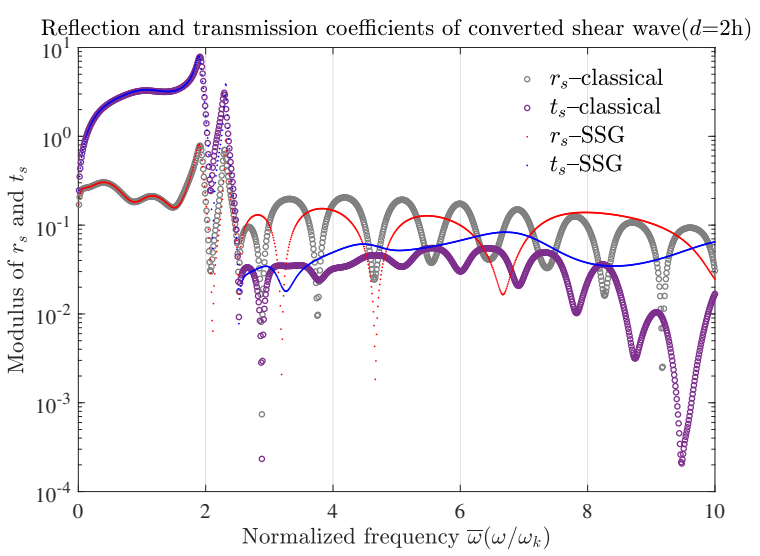

(d)

Figure 11: Transmission and reflection coefficients of bending wave through a complex beam made of $\mathrm{Cu}$

Figure 11 illustrates the transmission and reflection coefficient of bending wave $\left(t_{b}, r_{b}\right)$ and shear wave $\left(t_{s}, r_{s}\right)$ through a complex beam made of copper with the length $d=h$ and $d=2 h$. Noticeably, the scattering coefficients resulting from complex coupling region and the classical coupling region match well in the low-frequency range, but their difference becomes significant as frequency goes up, especially above the cut-on frequency of the shear wave. By comparing subfigure 11(a) and 11(b) with subfigure 11(c) and 11(d), we can see that wave transmission behavior changes significantly for both bending and shear wave as the coupling length $d$ increases from $h$ to $2 h$. The wave transmission behavior is affected by the dimension of the coupling region, and that is because the degree of nonlocal effect introduced by the higher-order material constants is dependent on the relative deformation wavelength (compared to the material characteristic length). The proposed higher gradient-based wave-scattering formulation is capable of capturing the local behavior caused by long-range interactions. The influence becomes more significant in the short wave limit.

\subsection{Control of vibration transmission through a complex beam}

For any kind of complex media which can be composites, architectured media, or metamaterials, when the deformation wavelength is comparable to the internal length scale, the long-range interaction between non-adjacent internal structures will play an important role in governing the structural global behavior. Similar to the investigation of the complex rod coupling region, the proposed SSG theory-based bending wave scattering formulation can be used in the application of wave transmission control through a complex beam by adjusting its higher-order material constants, which are equivalent to the stiffnesses of the short interaction and the long-range interactions. In the following, several 
numerical cases are implemented to study the influence of material parameters on bending wave transmission, with the purpose of obtaining the minimum possible energy reflection.

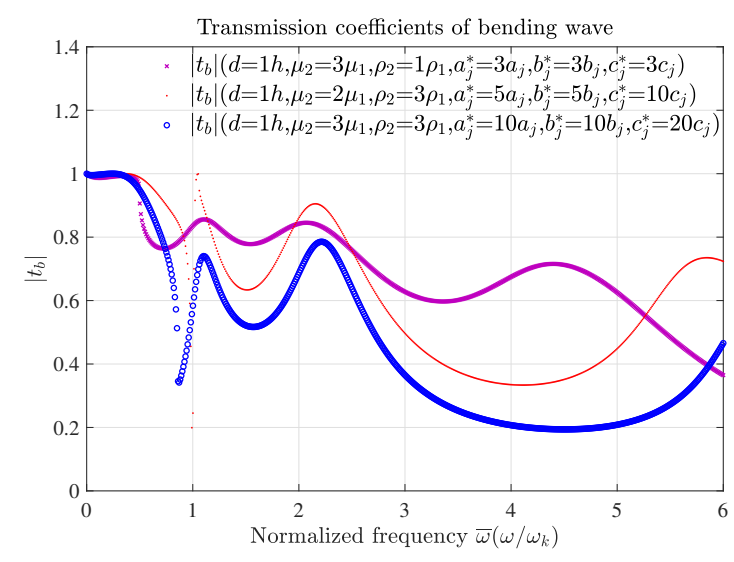

(a)

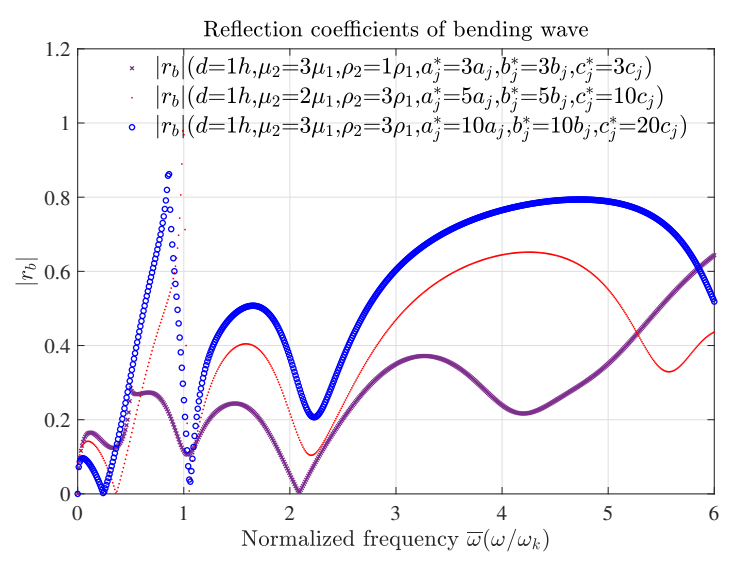

(b)

Figure 12: Transmission and reflection coefficients of bending wave through a complex beam in three cases (case 1: $\times$, case $2: \cdot$, case $3: \circ$ )

Figure 12 shows the transmission and reflection coefficients of bending wave $\left(t_{b} r_{b}\right)$ changing with different material parameters in three cases $\left(\mu_{1}, \rho_{1}, a_{j}, b_{j}, c_{j}\right.$ are the material parameters for aluminum). As we can see, for complex bean of length $d=h, t_{b}$ and $r_{b}$ change significantly in these three cases. In case $3, t_{b}$ highly approach ' 0 ' in higher frequency range, which means with some predefined shear modulus, mass density, and higher-order material parameters, the energy transmission by bending can approach to zero.

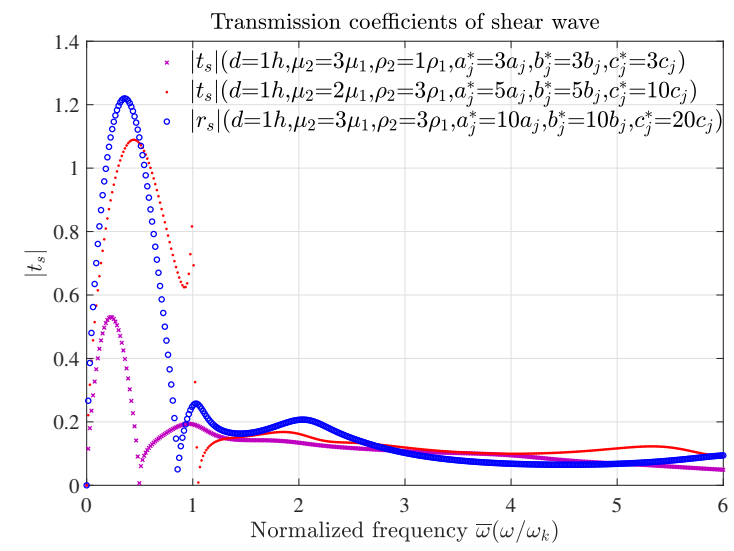

(a)

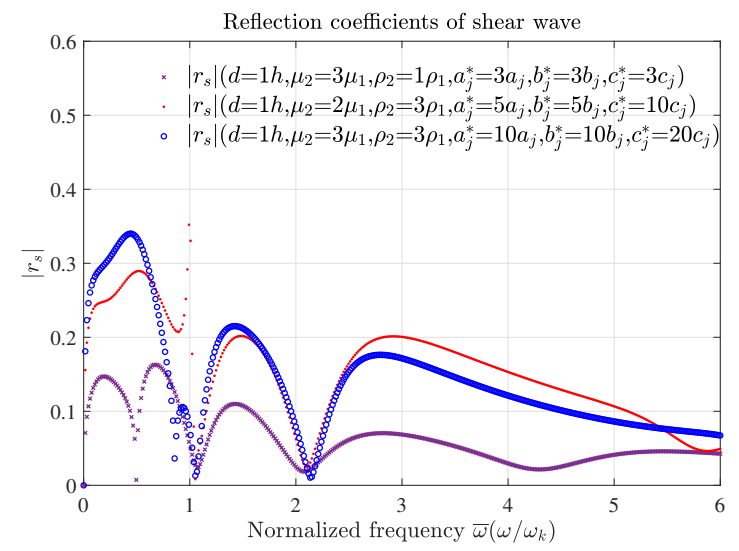

(b)

Figure 13: Transmission and reflection coefficients of the converted shear wave through a complex beam in three cases (case $1: \times$, case $2: \cdot$, case 3: ०)

Then we check the reflection and transmission of the converted shear waves. As shown in Figure 13, reflection and transmission of the shear wave are much weak than the bending wave. As the reflection of bending wave increase much and transmission decrease from case 1 to case 3 , the reflection of the shear wave only increases a little and the transmission rarely change. The impact of the non-local effect is less great for the shear wave than that of the bending wave. 


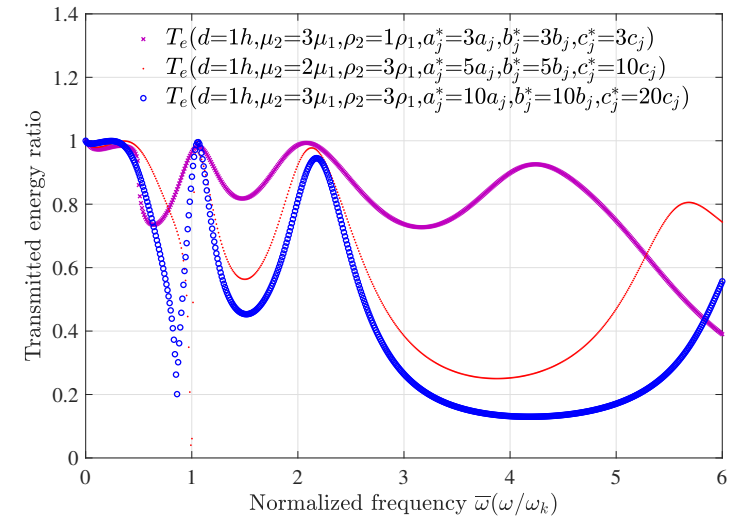

(a)

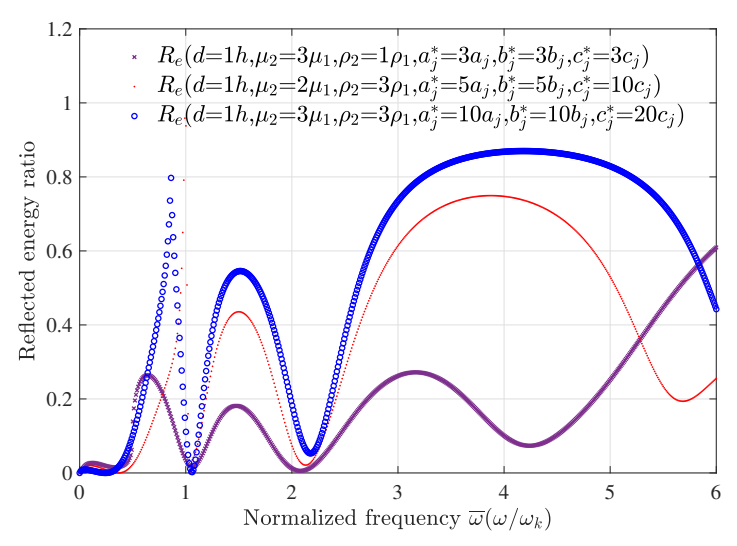

(b)

Figure 14: Transmitted and reflected energy through a complex beam in three cases (case $1: \times$, case $2: \bullet$, case $3: \circ)$

To check the energy transmitted situation, Figure 14 is plotted. It can be observed that energy transmission in case 3 is lower than 0.2 in frequency band $\bar{\omega} \in[3.2,5.2]$. In this frequency range, the complex beam in the coupling region shows great vibration isolation performance with the predefined material parameters, which are equivalent to the shortrange and long-range interactions between inner structures of periodic composite. The proposed formulation can be used for optimizing the inner micro structure of composites to achieve better structural performance in acoustics.

\section{Conclusions}

In this research, wave scattering characteristics of an enriched 1-D model are investigated based on SSG theory. The formulation is derived to achieve the Transmission and reflection coefficients of the longitudinal wave and bending wave through a complex rod and a complex Timoshenko beam, respectivly. An equivalent lattice model is proposed to study the underlying physical interpretation of higher-order material constants and to exploit the SSG theory formulation for potential applications to vibration transmission control.

(1) Wave transmission characteristics of a 1-D enriched model are different from the classical ones. They show frequency dependence for both rod and beam cases, especially at higher frequencies. The influence of local behavior caused by long-range interaction can be captured by the proposed model, and the non-local effects plays a major role in governing structural vibro-acoustic features under short wavelength deformation.

(2) As the length of the coupling region increase, SSG theory-based results are degenerating into classical results for both rod and beam cases. This phenomenon is caused not only by the longer propagation distance but also by the different wave propagation behaviors in the complex region. The non-local effect, which reflects the long-range interactions between the internal heterogeneity, becomes weak under a relatively longer deformation wavelength, and the resulting impedance mismatch becomes less obvious in global behavior description.

(3) The proposed lattice model is capable of describing the long-range interactions between the internal structures in complex media. With this lattice, higher-order material constants in SSG theory are directly connected with the stiffness of long-range interactions between the internal structures. Vibration transmission through complex regions can be controlled by optimizing its length and its inner micro-structure. Therefore SSG theory-based wave-scattering matrix model can be employed in optimizing the vibration transmission through complex media by adjusting its highorder material constants.

\section{Acknowledgements}

This work was supported by the LabEx CeLyA (Centre Lyonnais d'Acoustique, ANR-10-LABX-0060) of Université de Lyon. The research of G. Zhu is funded by a China Scholarship Council (CSC). The research of C. Droz is funded by the European Commission (WIDEA) under the grant agreement No. 797034. 


\section{Appendix 1}

To obtain the continuum equation from a lattice equation, a series of equation transformations is used here based on the map operation in [30]. Firstly, $u_{n}(t)$ are defined as Fourier coefficients of function $\hat{u}(k, t)$ on $\left[-K_{0} / 2, K_{0} / 2\right]$ by the equation

$$
\hat{u}(k, t)=\sum_{n=-\infty}^{+\infty} u_{n}(t) e^{-i k x_{n}}=\mathcal{F}_{\Delta}\left\{u_{n}(t)\right\},
$$

where $x_{n}=n l$ and $l=2 \pi / K_{0}$ is the distance between equilibrium positions of the lattice particles. The inverse Fourier series transform is defined by

$$
u_{n}(t)=\frac{1}{K_{0}} \int_{-K_{0} / 2}^{K_{0} / 2} d k \hat{u}(k, t) e^{i k x_{n}}=\mathcal{F}_{\Delta}^{-1}\{\hat{u}(k, t)\} .
$$

Equations (54) and (55) are the basis for the Fourier transform, which is obtained by transforming from lattice variable to a continuum one in the limit $l \rightarrow 0\left(K_{0}=\frac{2 \pi}{l} \rightarrow \infty\right)$. The Fourier transform are derived from (54) and (55) with limit $l \rightarrow 0$. We replace the lattice function $u_{n}(t)=\frac{2 \pi}{K_{0}} u\left(x_{n}, t\right)$ with continuous $u(x, t)$ while letting $x_{n}=n l=\frac{2 \pi n}{K_{0}} \rightarrow x$. Then the sum in Eq. 54 can be changed to an integral with $(l \rightarrow 0)$, and Eq. 54, 55) become

$$
\begin{aligned}
& \tilde{u}(k, t)=\int_{-\infty}^{+\infty} d x e^{-i k x} u(x, t)=\mathcal{F}\{u(x, t)\}, \\
& u(x, t)=\frac{1}{2 \pi} \int_{-\infty}^{+\infty} d k e^{i k x} \tilde{u}(k, t)=\mathcal{F}^{-1}\{\tilde{u}(k, t)\} .
\end{aligned}
$$

We assume that $\tilde{u}(k, t)=\lim \hat{u}(k, t)$, where limit denotes the passage to the limit $l \rightarrow 0\left(K_{0}=\frac{2 \pi}{l} \rightarrow \infty\right)$. As we can see, $\tilde{u}(k, t)$ is a Fourier transform of the field $u(x, t)$, and $\hat{u}(k, t)$ is a Fourier series transform of $u_{n}(t)$, where we can use $u_{n}(t)=\frac{2 \pi}{K_{0}} u(n l, t)$, and the function $\tilde{u}(x, t)$ can be derived from $\hat{u}(k, t)$ in the limit $l \rightarrow 0$. As a result, the map operation from a lattice model into a continuum model is defined by the following steps [32]

(1) The Fourier series transform:

$$
\mathcal{F}_{\Delta}: \quad u_{n}(t) \rightarrow \mathcal{F}_{\Delta}\left\{u_{n}(t)\right\}=\hat{u}(k, t)
$$

(2) The passage to the limit $l \rightarrow 0$ :

$$
\text { Limit }: \quad \hat{u}(k, t) \rightarrow \lim \{\hat{u}(k, t)\}=\tilde{u}(k, t)
$$

(3)The inverse Fourier transform:

$$
\mathcal{F}^{-1}: \quad \tilde{u}(k, t) \rightarrow \mathcal{F}^{-1}\{\tilde{u}(k, t)\}=u(x, t)
$$

The similar transformations can be performed for different systems to map the lattice equation into an equation for the elastic continuum. In the following, the transformations is proceeded based on the introduced map operation. The first step is transforming the discrete lattice equation of motion $u_{n}(t)$ to the field $\hat{u}(k, t)$. We multiply Eq. (49) by $e^{-i k n l}$, and summing over $n$ from $-\infty$ to $+\infty$. Then

$$
\begin{aligned}
M \sum_{n=-\infty}^{+\infty} e^{-i k n l} \frac{d^{2} u_{n}}{d t^{2}}= & g_{2} \cdot \sum_{n=-\infty}^{+\infty} e^{-i k n l}\left(u_{n+1}-2 u_{n}+u_{n-1}\right) \\
& +g_{4} \cdot \sum_{n=-\infty}^{+\infty} e^{-i k n l}\left(u_{n+2}-2 u_{n}+u_{n-2}\right) \\
& +g_{6} \cdot \sum_{n=-\infty}^{+\infty} e^{-i k n l}\left(u_{n+3}-2 u_{n}+u_{n-3}\right)+\sum_{n=-\infty}^{+\infty} e^{-i k n l} F(n) .
\end{aligned}
$$


The first three terms on the right-hand of Eq. 57] equals

$$
\begin{aligned}
= & g_{2} \cdot\left(e^{i k l} \sum_{m=-\infty}^{+\infty} e^{-i k m l} u_{m}-2 \sum_{n=-\infty}^{+\infty} e^{-i k n l} u_{n}+e^{-i k l} \sum_{j=-\infty}^{+\infty} e^{-i k j l} u_{j}\right) \\
& +g_{4} \cdot\left(e^{2 i k l} \sum_{m=-\infty}^{+\infty} e^{-i k m l} u_{m}-2 \sum_{n=-\infty}^{+\infty} e^{-i k n l} u_{n}+e^{-2 i k l} \sum_{j=-\infty}^{+\infty} e^{-i k j l} u_{j}\right) \\
& +g_{6} \cdot\left(e^{3 i k l} \sum_{m=-\infty}^{+\infty} e^{-i k m l} u_{m}-2 \sum_{n=-\infty}^{+\infty} e^{-i k n l} u_{n}+e^{-3 i k l} \sum_{j=-\infty}^{+\infty} e^{-i k j l} u_{j}\right) .
\end{aligned}
$$

Using the definition of $\hat{u}(k, t)$, Eq. (58) gives

$$
\begin{aligned}
& g_{2} \cdot\left(e^{i k l} \hat{u}(k, t)-2 \hat{u}(k, t)+e^{-i k l} \hat{u}(k, t)\right)+g_{4} \cdot\left(e^{2 i k l} \hat{u}(k, t)-2 \hat{u}(k, t)+e^{-2 i k l} \hat{u}(k, t)\right) \\
& +g_{6} \cdot\left(e^{3 i k l} \hat{u}(k, t)-2 \hat{u}(k, t)+e^{-3 i k l} \hat{u}(k, t)\right) \\
& =2\left(g_{2} \cdot(\cos (k l)-1)+g_{4} \cdot(\cos (2 k l)-1)+g_{6} \cdot(\cos (3 k l)-1)\right) \hat{u}(k, t) .
\end{aligned}
$$

To further simplify Eq. (59), we have

$$
\begin{aligned}
& \cos (k l)-1=-2 \sin ^{2}\left(\frac{k l}{2}\right) ; \cos (2 k l)-1=-8 \sin ^{2}\left(\frac{k l}{2}\right)+8 \sin ^{4}\left(\frac{k l}{2}\right) \\
& \cos (3 k l)-1=-32 \sin ^{6}\left(\frac{k l}{2}\right)-18 \sin ^{2}\left(\frac{k l}{2}\right)+48 \sin ^{4}\left(\frac{k l}{2}\right) .
\end{aligned}
$$

Substitution of Eq. (60) into Eq. (59) gives

$$
=-4\left(g_{2}+4 g_{4}+9 g_{6}\right) \sin ^{2}\left(\frac{k l}{2}\right) \hat{u}(k, t)+16\left(g_{4}+6 g_{6}\right) \sin ^{4}\left(\frac{k l}{2}\right) \hat{u}(k, t)-64 g_{6} \sin ^{6}\left(\frac{k l}{2}\right) \hat{u}(k, t) .
$$

With the obtained first three-terms equation and using the definition of $\hat{u}(k, t)$ for the other two terms, 57) becomes

$$
\begin{aligned}
M \frac{\partial^{2} \hat{u}(k, t)}{\partial t^{2}}= & -4\left(g_{2}+4 g_{4}+9 g_{6}\right) \sin ^{2}\left(\frac{k l}{2}\right) \hat{u}(k, t)+16\left(g_{4}+6 g_{6}\right) \sin ^{4}\left(\frac{k l}{2}\right) \hat{u}(k, t) \\
& -64 g_{6} \sin ^{6}\left(\frac{k l}{2}\right) \hat{u}(k, t)+\mathcal{F}\{F(n)\} .
\end{aligned}
$$

Using the asymptotic behavior of the sine in the form

$$
\sin \left(\frac{k l}{2}\right)=\frac{k l}{2}-\frac{1}{6}\left(\frac{k l}{2}\right)^{3}+\frac{1}{5 !}\left(\frac{k l}{2}\right)^{5}+O\left((k l)^{7}\right)
$$

and replacing the terms of $\sin ^{2}\left(\frac{k l}{2}\right), \sin ^{4}\left(\frac{k l}{2}\right)$ and $\sin ^{6}\left(\frac{k l}{2}\right)$ in Eq. 61) gives

$$
=-4\left(g_{2}+4 g_{4}+9 g_{6}\right)(k l)^{2}+\frac{1}{12}\left(g_{2}+16 g_{4}+81 g_{6}\right)(k l)^{4}-\frac{1}{360}\left(g_{2}+64 g_{4}+729 g_{6}\right)(k l)^{6} .
$$

The transition to the limit $d \rightarrow 0$ in Eq. (61) gives

$$
\frac{\partial^{2} \tilde{u}(k, t)}{\partial t^{2}}=-G_{2} k^{2} \tilde{u}(k, t)+G_{4} k^{4} \tilde{u}(k, t)-G_{6} k^{6} \tilde{u}(k, t)+\mathcal{F}\{F(x)\},
$$

where

$$
G_{2}=\frac{\left(g_{2}+4 g_{4}+9 g_{6}\right) l^{2}}{M}, G_{4}=\frac{\left(g_{2}+16 g_{4}+81 g_{6}\right) l^{4}}{12 M}, G_{6}=\frac{\left(g_{2}+64 g_{4}+729 g_{6}\right) l^{6}}{360 M}
$$


The inverse Fourier transform $\mathcal{F}^{-1}$ of Eq. (64) has the form

$$
\frac{\partial^{2} \mathcal{F}^{-1}\{\tilde{u}(k, t)\}}{\partial t^{2}}=-G_{2} \mathcal{F}^{-1}\left\{k^{2} \tilde{u}(k, t)\right\}+G_{4} \mathcal{F}^{-1}\left\{k^{4} \tilde{u}(k, t)\right\}-G_{6} \mathcal{F}^{-1}\left\{k^{6} \tilde{u}(k, t)\right\}+\frac{1}{\rho} f(x) .
$$

Then we can use the relation $\mathcal{F}^{-1}\{\tilde{u}(k, t)\}=u(x, t)$ and the connection between the derivatives and its Fourier transforms as

$$
F^{-1}\left\{k^{2} \tilde{u}(k, t)\right\}=-\frac{\partial^{2} u(x, t)}{\partial x^{2}}, F^{-1}\left\{k^{4} \tilde{u}(k, t)\right\}=+\frac{\partial^{4} u(x, t)}{\partial x^{4}}, F^{-1}\left\{k^{6} \tilde{u}(k, t)\right\}=-\frac{\partial^{6} u(x, t)}{\partial x^{6}} .
$$

Then the continuum equation can be achieved as

$$
\frac{\partial^{2} u(x, t)}{\partial t^{2}}=G_{2} \frac{\partial^{2} u(x, t)}{\partial x^{2}}+G_{4} \frac{\partial^{4} u(x, t)}{\partial x^{4}}+G_{6} \frac{\partial^{6} u(x, t)}{\partial x^{6}}+F(x),
$$

\section{References}

[1] X. Liu, J. Banerjee, Free vibration analysis for plates with arbitrary boundary conditions using a novel spectral-dynamic stiffness method Computers \& Structures 164 (2016) 108-126. doi:https://doi.org/10.1016/j.compstruc.2015.11.005 URL https://www.sciencedirect.com/science/article/pii/S0045794915003028

[2] E. El Masri, N. Ferguson, T. Waters, Wave propagation and scattering in reinforced concrete beams, The Journal of the Acoustical Society of America 146 (5) (2019) 3283-3294. doi:10.1121/1.5131644

[3] F. Shirmohammadi, S. Bahrami, M. M. Saadatpour, A. Esmaeily, Modeling wave propagation in moderately thick rectangular plates using the spectral element method Applied Mathematical Modelling 39 (12) (2015) 3481-3495. doi: https://doi.org/10.1016/j .apm.2014. 11.044

URL https://www.sciencedirect.com/science/article/pii/S0307904X14006398

[4] U. Lee, Spectral Element Method in Structural Dynamics, John Wiley \& Sons (Asia), Singapore, 2009. doi:10.1002/9780470823767

[5] N. J. Kessissoglou, Power transmission in 1-shaped plates including flexural and in-plane vibration The Journal of the Acoustical Society of America 115 (3) (2004) 1157-1169. doi:10.1121/1.1635415

URL https://doi.org/10.1121/1.1635415

[6] V. COTONI, A. LE BOT, L. JEZEQUEL, High-frequency radiation of 1-shaped plates by a local energy flow approach Journal of Sound and Vibration 250 (3) (2002) 431-444. doi:https://doi.org/10.1006/jsvi.2001.3952

URL https://www.sciencedirect.com/science/article/pii/S0022460X01939525

[7] M. Ichchou, J.-M. Mencik, W. Zhou, Wave finite elements for low and mid-frequency description of coupled structures with damage, Computer methods in applied mechanics and engineering 198 (15-16) (2009) 1311-1326.

[8] C. Droz, O. Bareille, J.-P. Lainé, M. N. Ichchou, Wave-based shm of sandwich structures using cross-sectional waves, Structural Control and Health Monitoring 25 (2) (2018) e2085.

[9] N. Nanda, S. Kapuria, S. Gopalakrishnan, Spectral finite element based on an efficient layerwise theory for wave propagation analysis of composite and sandwich beams Journal of Sound and Vibration 333 (14) (2014) 3120-3137. doi:https://doi .org/10.1016/j.jsv. 2014.02 .036

URL http://www.sciencedirect.com/science/article/pii/S0022460X14001771

[10] J. Reddy, Nonlocal theories for bending, buckling and vibration of beams, International Journal of Engineering Science 45 (2-8) (2007) 288-307. doi:10.1016/j.ijengsci.2007.04.004

[11] J. N. Reddy, S. D. Pang, Nonlocal continuum theories of beams for the analysis of carbon nanotubes Journal of Applied Physics 103 (2) (2008) 023511. doi:10.1063/1.2833431 URL https://doi.org/10.1063/1.2833431

[12] G. Tsiatas, A new kirchhoff plate model based on a modified couple stress theory. International Journal of Solids and Structures 46 (13) (2009) 2757 -2764. doi:https://doi.org/10.1016/j.ijsolstr.2009.03.004 URL http://www.sciencedirect.com/science/article/pii/S0020768309001036

[13] H. T. Thai, D. H. Choi, Size-dependent functionally graded kirchhoff and mindlin plate models based on a modified couple stress theory. Composite Structures 95 (2013) 142 - 153. doi:https://doi.org/10.1016/j.compstruct.2012.08.023 URL http://www.sciencedirect.com/science/article/pii/S0263822312003893

[14] S. Gopalakrishnan, Propagation of elastic waves in nanostructures, in: Nanosensors, Biosensors, and Info-Tech Sensors and Systems 2016, Vol. 9802, 2016, p. 98020N. doi:10.1117/12.2218203

[15] L. Li, Y. Hu, L. Ling, Flexural wave propagation in small-scaled functionally graded beams via a nonlocal strain gradient theory Composite Structures 133 (2015) 1079 - 1092. doi:https://doi.org/10.1016/j.compstruct.2015.08.014 URL http://www.sciencedirect.com/science/article/pii/S0263822315006911

[16] L. Placidi, G. Rosi, I. Giorgio, A. Madeo, Reflection and transmission of plane waves at surfaces carrying material properties and embedded in second-gradient materials Mathematics and Mechanics of Solids 19 (5) (2014) 555-578. doi:10.1177/1081286512474016 URL https://doi.org/10.1177/1081286512474016 
[17] Y. Li, P. Wei, Reflection and transmission of thermo-elastic waves without energy dissipation at the interface of two dipolar gradient elastic solids Journal of the Acoustical Society of America 143 (1) (2018) 550-562. doi : 10.1121/1.5020780

URL https://www . scopus. com/inward/record.uri?eid=2-s2.0-85041445768

[18] Y. Li, P. Wei, Q. Tang, Reflection and transmission of elastic waves at the interface between two gradient-elastic solids with surface energy. European Journal of Mechanics - A/Solids 52 (2015) 54-71. doi:https://doi.org/10.1016/j.euromechsol.2015.02.001 URL https://www.sciencedirect.com/science/article/pii/S0997753815000145

[19] G. Zhu, C. Droz, A. Zine, M. Ichchou, Wave propagation analysis for a second strain gradient rod theory Chinese Journal of Aeronauticsdoi : https://doi.org/10.1016/j.cja.2019.10.006

URL http://www.sciencedirect.com/science/article/pii/S1000936119304091

[20] C. Wang, X. Chen, P. Wei, Y. Li, Reflection and transmission of elastic waves through a couple-stress elastic slab sandwiched between two half-spaces, Acta Mechanica Sinica 33 (2017) 1022-1039. doi:https ://doi.org/10.1007/s10409-017-0712-5

[21] C. Liu, J. Yu, X. Zhang, B. Zhang, L. Elmaimouni, Reflection behavior of elastic waves in the functionally graded piezoelectric microstructures European Journal of Mechanics - A/Solids 81 (2020) 103955. doi:https://doi.org/10.1016/j .euromechsol.2020.103955 URL https://www.sciencedirect.com/science/article/pii/S099775381930662X

[22] R. D. Mindlin, Second gradient of strain and surface-tension in linear elasticity, International Journal of Solids and Structures 1 (4) (1965) 417 - 438. doi:https://doi.org/10.1016/0020-7683(65)90006-5

[23] M. Asghari, S. Momeni, R. Vatankhah, The second strain gradient theory-based timoshenko beam model, Journal of Vibration and Control 23 (13) (2017) 2155-2166. doi:10.1177/1077546315611822

[24] M. Kandaz, H. Dal, M. Ünlü, Analysis of gold microbeams with higher order continuum theories. Proc. Appl. Math. Mech. 17 (1) (2017) 421-422. doi:10.1002/pamm.201710180

URL https://onlinelibrary.wiley.com/doi/abs/10.1002/pamm.201710180

[25] H. Espinosa, B. Prorok, M. Fischer, A methodology for determining mechanical properties of freestanding thin films and mems materials Journal of the Mechanics and Physics of Solids 51 (1) (2003) 47 -67. doi : https://doi.org/10.1016/S0022-5096(02) 00062-5 URL http://www.sciencedirect.com/science/article/pii/S0022509602000625

[26] R. Ansari, R. Gholami, H. Rouhi, Vibration analysis of single-walled carbon nanotubes using different gradient elasticity theories Composites Part B: Engineering 43 (8) (2012) 2985 - 2989. doi : https://doi.org/10.1016/j . compositesb.2012.05.049 URL http://www.sciencedirect.com/science/article/pii/S1359836812003782

[27] A. V. Metrikine, H. Askes, An isotropic dynamically consistent gradient elasticity model derived from a $2 \mathrm{~d}$ lattice, Philosophical Magazine 86 (21-22) (2006) 3259-3286. doi:10.1080/14786430500197827

[28] D. De Domenico, H. Askes, E. C. Aifantis, Gradient elasticity and dispersive wave propagation: Model motivation and length scale identification procedures in concrete and composite laminates International Journal of Solids and Structures 158 (2019) 176-190. doi:https: //doi.org/10.1016/j.ijsolstr.2018.09.007

URLhttps://www.sciencedirect.com/science/article/pii/S0020768318303639

[29] D. De Domenico, H. Askes, Stress gradient, strain gradient and inertia gradient beam theories for the simulation of flexural wave dispersion in carbon nanotubes Composites Part B: Engineering 153 (2018) 285-294. doi:https://doi.org/10.1016/j.compositesb.2018. 08.083

URL https://www.sciencedirect.com/science/article/pii/S1359836818313635

[30] V. E. Tarasov, Lattice model with nearest-neighbor and next-nearest-neighbor interactions for gradient elasticity, Discontinuity, Nonlinearity, and Complexity 4 (1) (2015) 11-23. doi:10.5890/DNC.2015.03.002

[31] H. M. Shodja, F. Ahmadpoor, A. Tehranchi, Calculation of the additional constants for fcc materials in second strain gradient elasticity: Behavior of a nano-size bernoulli-euler beam with surface effects Journal of Applied Mechanics 79 (2). doi:10.1115/1.4005535 URL https://doi.org/10.1115/1.4005535

[32] V. E. Tarasov, Continuous limit of discrete systems with long-range interaction, Journal of Physics A: Mathematical and General 39 (48) (2006) 14895-14910. doi:10.1088/0305-4470/39/48/005 